ARTICLE

\title{
The FNIP co-chaperones decelerate the Hsp90 chaperone cycle and enhance drug binding
}

\author{
Mark R. Woodford 1,2, Diana M. Dunn 1,2,3, Adam R. Blanden 2,3, Dante Capriotti1,2, David Loiselle ${ }^{4}$, \\ Chrisostomos Prodromou ${ }^{5}$, Barry Panaretou 6 , Philip F. Hughes ${ }^{4}$, Aaron Smith ${ }^{4}$, Wendi Ackerman ${ }^{7}$, \\ Timothy A. Haystead ${ }^{4}$, Stewart N. Loh ${ }^{2,3}$, Dimitra Bourboulia1,2,3, Laura S. Schmidt ${ }^{8,9}$, W. Marston Linehan?, \\ Gennady Bratslavsky ${ }^{1,2} \&$ Mehdi Mollapour ${ }^{1,2,3}$
}

Heat shock protein-90 (Hsp90) is an essential molecular chaperone in eukaryotes involved in maintaining the stability and activity of numerous signalling proteins, also known as clients. Hsp90 ATPase activity is essential for its chaperone function and it is regulated by co-chaperones. Here we show that the tumour suppressor FLCN is an Hsp90 client protein and its binding partners FNIP1/FNIP2 function as co-chaperones. FNIPs decelerate the chaperone cycle, facilitating FLCN interaction with Hsp90, consequently ensuring FLCN stability. FNIPs compete with the activating co-chaperone Aha1 for binding to Hsp90, thereby providing a reciprocal regulatory mechanism for chaperoning of client proteins. Lastly, downregulation of FNIPs desensitizes cancer cells to Hsp90 inhibitors, whereas FNIPs overexpression in renal tumours compared with adjacent normal tissues correlates with enhanced binding of Hsp90 to its inhibitors. Our findings suggest that FNIPs expression can potentially serve as a predictive indicator of tumour response to Hsp9O inhibitors.

\footnotetext{
${ }^{1}$ Department of Urology, SUNY Upstate Medical University, 750 East Adams Street, Syracuse, New York 13210, USA. ${ }^{2}$ Cancer Research Institute, SUNY Upstate Medical University, 750 East Adams Street, Syracuse, New York 13210, USA. ${ }^{3}$ Department of Biochemistry and Molecular Biology, SUNY Upstate Medical University, 750 East Adams Street, Syracuse, New York 13210, USA. ${ }^{4}$ Department of Pharmacology and Cancer Biology, Duke University Medical Center, Durham, North Carolina 27710, USA. ${ }^{5}$ Genome Damage and Stability Centre, University of Sussex, Brighton BN1 9RQ, UK. ${ }^{6}$ Institute of Pharmaceutical Science, King's College London, London SE1 9NH, UK. ${ }^{7}$ Health Sciences Library, SUNY Upstate Medical University, 750 East Adams Street, Syracuse, New York 13210, USA. ${ }^{8}$ Basic Science Program, Leidos Biomedical Research, Inc., Frederick National Laboratory for Cancer Research, Frederick, Maryland 21702, USA. ${ }^{9}$ Urologic Oncology Branch, Center for Cancer Research, National Cancer Institute, 9000 Rockville Pike, Bethesda, Maryland 20892, USA. Correspondence and requests for materials should be addressed to M.M. (email: mollapom@upstate.edu).
} 
T he molecular chaperone heat shock protein-90 (Hsp90) is responsible for folding, stability and activity of many proteins also known as 'client proteins', including many responsible for tumour initiation, progression and metastasis ${ }^{1}$. This makes the chaperone Hsp90 an attractive target for cancer therapy ${ }^{2}$. Hsp90 has the ability to bind and hydrolyse ATP, which is essential for its chaperone function ${ }^{3}$. Small molecule inhibitors bind to the ATP-binding pocket of $\mathrm{Hsp} 90$ and inhibit its chaperone function. Consequently, this prevents Hsp90 interaction with client proteins, leading to their degradation by the proteasome. In contrast to other anticancer drugs, Hsp90 inhibitors simultaneously inhibit multiple drivers of oncogenesis.

Hsp90 chaperone cycle is tightly regulated by another group of proteins referred to as 'co-chaperones'. Their stability does not depend on Hsp90 function but they interact with distinct Hsp90 conformational states, providing directionality to the Hsp90 cycle $^{4}$. Furthermore, certain co-chaperones, such as HOP and Cdc $37^{\mathrm{p} 50}$ inhibit the Hsp90 chaperone cycle, assisting in delivery of distinct sets of client proteins (steroid hormone receptors and kinases, respectively) to the Hsp90 chaperone machine. In contrast, the co-chaperone Ahal facilitates energy-intensive conformational changes necessary to establish Hsp90 ATPase competence, markedly increasing the weak endogenous ATPase activity of Hsp90. Ahal is thus considered to be a crucial component of active Hsp90 chaperone complexes ${ }^{5,6}$.

Here we show that the stability of the tumour suppressor folliculin (FLCN) depends on the chaperone function of Hsp90. Germline mutations and loss of function of FLCN causes BirtHogg-Dubé syndrome, a rare inherited cancer syndrome that predisposes affected individuals to develop kidney tumours, pulmonary cysts and benign skin tumours (fibrofolliculomas). FLCN interacts and forms a complex with folliculin-interacting proteins 1 and 2 (FNIP1 and FNIP2, also referred to as FNIPs) ${ }^{8-10}$. The function of FNIPs, however, remains elusive. Our results indicate that FNIPs act as co-chaperones of Hsp90. They inhibit its ATPase activity, 'tailoring' Hsp90 to chaperone kinase and non-kinase clients. We have further shown that Ahal co-chaperone can displace FNIPs and stimulate Hsp90 ATPase activity. Finally, FNIPs also enhance the binding of Hsp90 to its inhibitors such as ganetespib (GB); therefore, overexpression of FNIPs in specific tumours can be an indicator of their response to Hsp90 inhibitors.

\section{Results}

FLCN is a new client of Hsp90. To determine the binding partners of the tumour suppressor FLCN, we transiently expressed an amino-terminally FLAG-tagged FLCN (FLAGFLCN) in human embryonic kidney 293 (HEK293) cells and identified its intracellular binding proteins by immunoprecipitating FLAG-FLCN with anti-FLAG M2 affinity gel and mass spectrometry (MS) analysis (Fig. 1a and Supplementary Table 1). We found molecular chaperones heat shock protein-70 (Hsp70) and Hsp90, and their regulators HOP, CHIP and Ahal, and CCT2, CCT4, CCT7 and CCT8, which are members of the chaperonin system TRiC (TCP-1 ring complex), (Fig. 1a). We validated our data by immunoprecipitating the endogenous FLCN (Fig. 1b) or the FLAG-FLCN (Fig. 1c) from HEK293 cells and showed its interaction with the molecular chaperone machineries Hsp70, Hsp90 and a subunit of the chaperonin TRiC, CCT2 (Fig. 1b,c). We also observed FLCN interaction with the Hsp70 and Hsp90 co-chaperones including HOP, CHIP, Cdc $37^{\mathrm{p} 50}$, PP5, p23 and Aha1 (Fig. 1b,c). In general, molecular chaperones are involved in folding and stability of proteins. We first treated the HEK293 cells with the Hsp70 inhibitor JG-98 (ref. 11) and showed the degradation of FLCN after a $2 \mathrm{~h}$ treatment in both soluble and insoluble protein fractions (Fig. 1d). These data suggest that inhibition of Hsp70 does not lead to an increase in misfolded FLCN but instead to its degradation. The molecular chaperone Hsp90 however is more selective towards its 'client proteins' and is also involved in protecting them from degradation ${ }^{12}$. Therefore, we treated the HEK293 cells with different inhibitors of Hsp90 such as GB ${ }^{13}$ (Fig. 1e), SNX2112 (ref. 14) and PU-H71 (ref. 15) (Supplementary Fig. 1a,b), to evidence the degradation of FLCN. Previous works have shown that inhibition of Hsp90 generally leads to ubiquitination and degradation of its client proteins in the proteasome ${ }^{16}$. We investigated this possibility by first demonstrating that inhibition of Hsp90 causes its dissociation from FLCN (Fig. 1f). We further showed that HEK293 cells treated with $50 \mathrm{nM}$ proteasome inhibitor bortezomib for $2 \mathrm{~h}$ before addition of GB blocked FLCN degradation (Fig. 1g). We did not obtain similar results when we treated the cells with the lysosomal inhibitor Bafilomycin A1 (Supplementary Fig. 1c), suggesting that the lysosome is not involved in degradation of FLCN. We next immunoprecipitated and salt-stripped (with $0.5 \mathrm{M} \mathrm{NaCl}$ ) FLAG-FLCN from HEK293 cells treated with either $50 \mathrm{nM}$ bortezomib or $1 \mu \mathrm{M}$ GB for $4 \mathrm{~h}$ and showed its ubiquitination by western blotting (Fig. 1h). Taken together, our results suggest that FLCN is a client of Hsp70 and Hsp90. Inhibition of Hsp90 leads to ubiquitination and degradation of FLCN in the proteasome.

FNIP1 and FNIP2 facilitate FLCN binding to Hsp90 chaperone. FNIP1 and FNIP2 are homologous binding partners of FLCN 8,10 ; however, their molecular function remains elusive. In addition, FNIP1 was shown to interact with Hsp90, but the significance of this observation was not researched further ${ }^{8}$. We first confirmed these data by immunoprecipitating the endogenous FNIP1 and FNIP2 from HEK293 cells and detecting Hsp90 (Fig. 2a). We also observed FNIPs interaction with Hsp70 and co-chaperones p23, HOP and $\mathrm{Cdc} 37^{\mathrm{p} 50}$ (Fig. 2a). In addition, we co-immunoprecipitated Hsp90 clients such as glucocorticoid receptor (GR), B-Raf, Cdk4 and FLCN (Fig. 2a). We confirmed our data by transiently expressing and immunoprecipitating HA-FNIP1 and HA-FNIP2 from HEK293 cells and then probing for the chaperones, co-chaperone and client proteins (Supplementary Fig. 2a). Previous work has shown that FNIPs are homo- and heterodimer proteins ${ }^{10}$. We isolated FNIP1 and FNIP2 homo- and heterodimers by transiently co-expressing cMyc-FNIP1/HA-FNIP1, cMyc-FNIP2/HAFNIP2 and HA-FNIP1/cMyc-FNIP2 in HEK293 cells. We first immunoprecipitated cMyc-tagged proteins using anti-cMyc agarose affinity gel. We then competed the $\mathrm{cMyc}$ proteins from the affinity gel with $\mathrm{cMyc}$ peptides. The eluted proteins were dialysed and subjected to a second round of immunoprecipitation (IP) using anti-HA agarose affinity gel. Immunoprecipitated proteins have shown that FNIP1 homodimer has a stronger affinity for binding to Hsp90 and FLCN than FNIP2 (Fig. 2b). We next asked whether FNIP1 or FNIP2 are clients or co-chaperones of Hsp90. Treating HEK293 cells with the Hsp90 inhibitor GB did not affect FNIP protein stability (Fig. 2c). These data suggest that FNIP1 and FNIP2 are co-chaperones of Hsp90. We obtained further evidence by demonstrating a direct interaction between FNIP1 and Hsp90. We were able to bacterially express and purify a small amount of FLAG-FNIP1. Our purified FLAG-FNIP1 can directly interact with bacterially expressed and purified Hsp90 $\alpha-\mathrm{His}_{6}$ (Fig. 2d) and $\mathrm{FLCN}-\mathrm{His}_{6}$ (Fig. 2e). Surprisingly, Hsp90 $\alpha$ and FLCN did not directly interact with each other in vitro; however, pre-incubation of $\mathrm{Hsp} 90 \alpha$ with FNIP1 facilitated the Hsp90-FNIP1-FLCN complex 
a
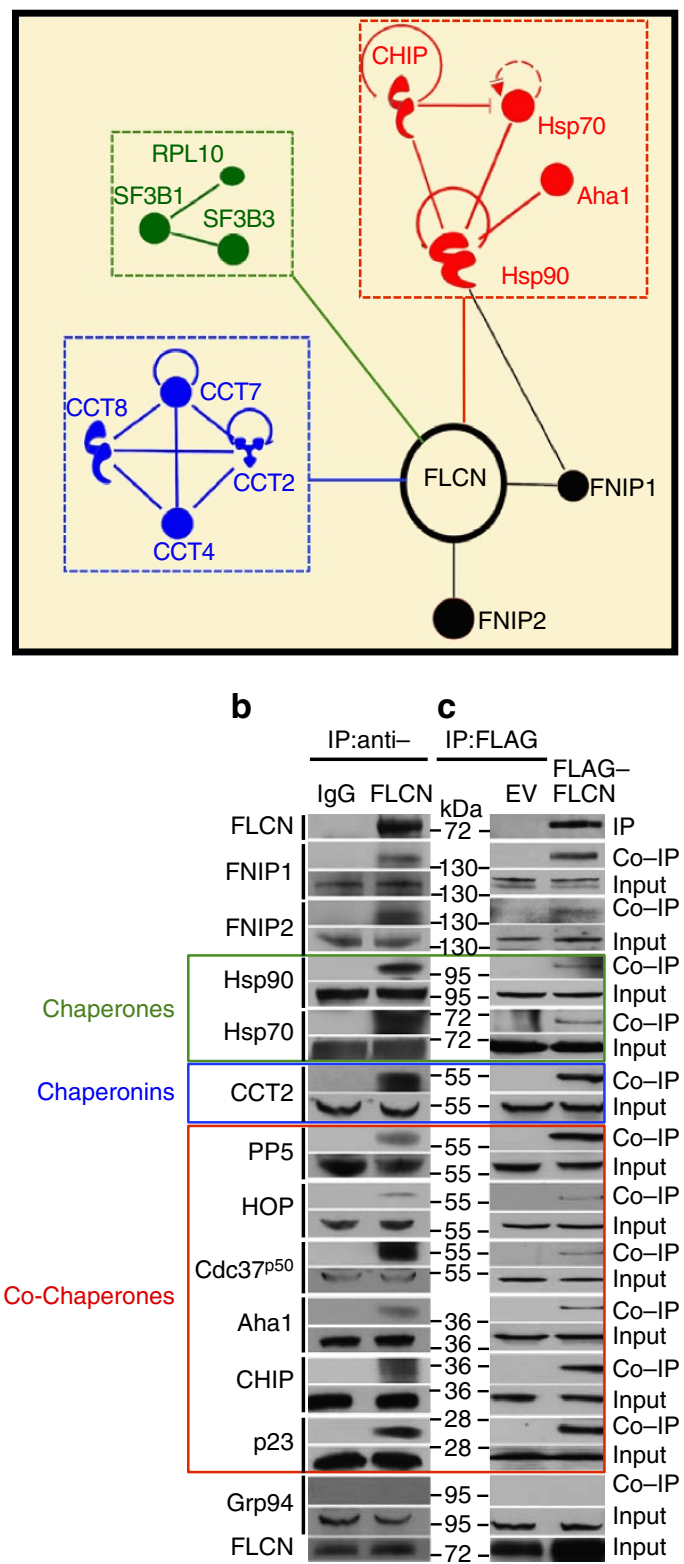

d

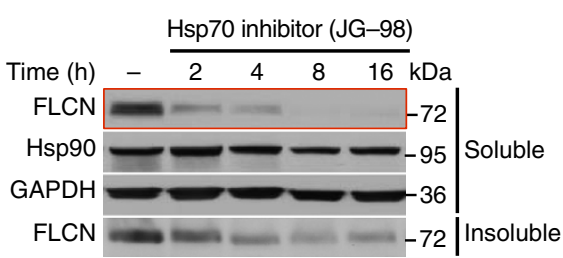

e

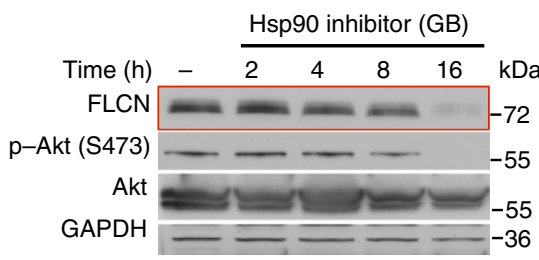

f

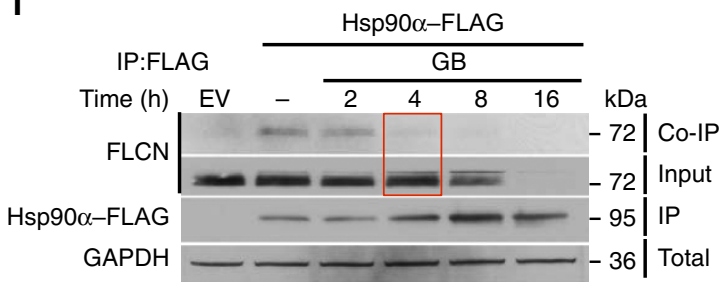

g

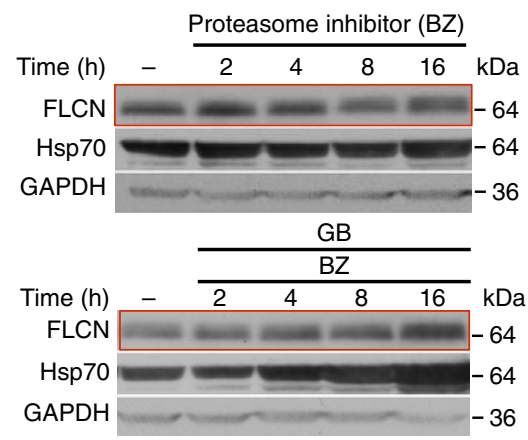

h

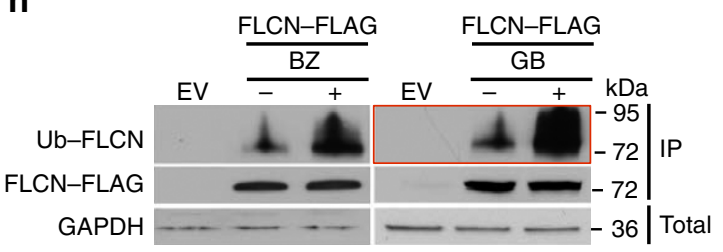

Figure 1 | Folliculin is a new client of Hsp90. (a) FLAG-FLCN was expressed and isolated from HEK293 cells. Profile of interacting proteins determined by MALDI-time of flight. Red nodes represent chaperones and co-chaperones, blue nodes are chaperonins and green nodes are splicing factors and ribosomal proteins. (b) FLCN was isolated from HEK293 cell lysates using anti-FLCN or lgG (control) and immunoblotted with indicated antibodies to confirm protein interactions. (c) HEK293 cells were transiently transfected with FLAG-FLCN or empty vector control (EV), immunoprecipitated and immunoblotted with indicated antibodies to confirm interacting proteins. (d) HEK293 cells were treated with $10 \mu \mathrm{M}$ of the Hsp70 inhibitor JG-98 at the indicated time points. FLCN protein stability in soluble and insoluble fraction was assessed by immunoblotting. (e) HEK293 cells were treated with $1 \mu \mathrm{M}$ GB at the indicated time points. FLCN protein stability was assessed by immunoblotting. Akt and Phospho-S473-Akt were used as positive controls. (f) Hsp90 $\alpha$-FLAG was transiently expressed in HEK293 cells. Cells were treated with $1 \mu \mathrm{M} \mathrm{GB}$ for the indicated times. Hsp90 $\alpha$-FLAG was immunoprecipitated and co-IP of FLCN was examined by immunoblotting. (g) HEK293 cells were treated with $50 \mathrm{nM}$ of the proteasome inhibitor bortezomib (BZ) for the indicated times. FLCN protein levels were evaluated at the indicated time points by immunoblotting (upper blots). HEK293 cells were also treated with $1 \mu \mathrm{M}$ GB for $1 \mathrm{~h}$ before addition of $50 \mathrm{nM} \mathrm{BZ}$. Immunoblotting was used to evaluate the FLCN level for the indicated time points (lower blots). (h) Empty vector (EV) or FLAGFLCN was used to transiently transfect HEK293 cells for $24 \mathrm{~h}$ then treated for $4 \mathrm{~h}$ with either $50 \mathrm{~nm}$ BZ or $1 \mu \mathrm{M}$ GB. FLAG-FLCN was immunoprecipitated and ubiquitination was examined by immunoblotting with a pan-anti-ubiquitin antibody.

formation (Fig. 2f). It is noteworthy that we were unable to express and purify an adequate amount of FNIP2 protein from bacteria, yeast or baculovirus expression systems for biophysical analysis.
Hsp90 consists of $\mathrm{N}$ (amino), $\mathrm{M}$ (middle) and $\mathrm{C}$ (carboxy) domains. To determine FNIP and FLCN interaction with these Hsp90 domains, we transiently expressed each domain with FLAG-tag in HEK293 cells. Following IP with anti-FLAG M2 
a

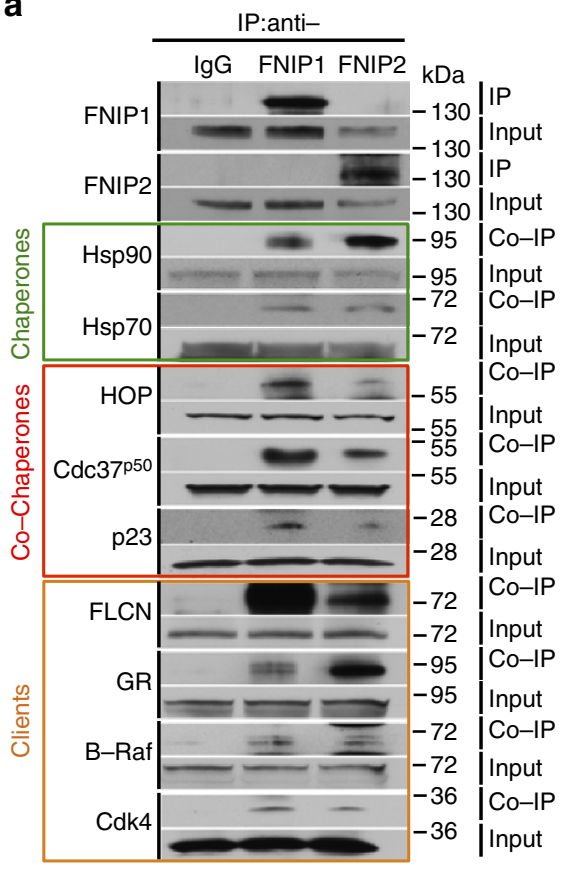

b

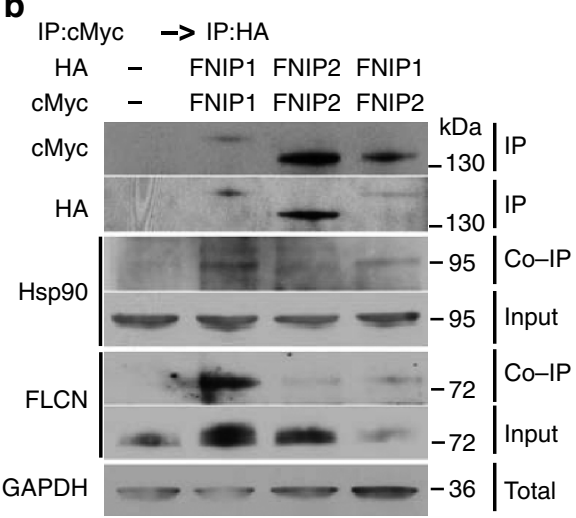

C

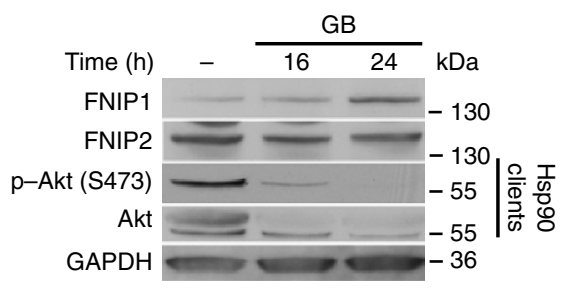

d



e
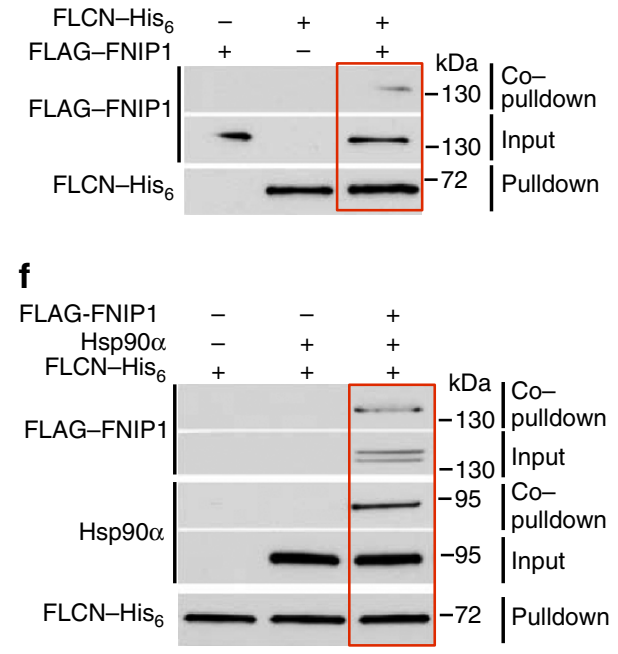

Figure 2 | FNIPs facilitate FLCN binding to the Hsp90 chaperone. (a) FNIP1 and FNIP2 were isolated from HEK293 cell lysates using anti-FNIP1, antiFNIP2 or lgG (control) and immunoblotted with indicated antibodies to confirm protein interaction. (b) HA-FNIP1 and HA-FNIP2 were transiently co-expressed with either cMyc-FNIP1 or cMyc-FNIP2 in HEK293 cells. cMyc-FNIP1 and cMyc-FNIP2 were first isolated followed by IP of HA-FNIP1 and HA-FNIP2 from the same samples. The quality of HA-FNIP1:cMyc-FNIP1 and HA-FNIP2:cMyc-FNIP2 homodimers and HA-FNIP1:cMyc-FNIP2 heterodimer were assessed by immunoblotting. Co-IP of Hsp90 and FLCN was also assessed by western blotting. (c) HEK293 cells were treated with $1 \mu \mathrm{M}$ GB for the indicated times, and FNIP1 and FNIP2 protein levels were determined by immunoblotting. Akt and Phospho-S473-Akt were used as positive controls. (d) FLAG-FNIP1 interacts with $\mathrm{Hsp}_{90} \alpha-\mathrm{His}_{6}$ in vitro. Bacterially expressed and purified $\mathrm{Hsp} 90 \alpha-\mathrm{His}_{6}$ was bound to Ni-NTA agarose and then incubated with 10 ng pure FLAG-FNIP1. Hsp90 $\alpha$-His ${ }_{6}$ pulldown and FLAG-FNIP1 co-pulldown were assessed by immunoblotting. (e) FLAG-FNIP1 interacts with FLCN-His ${ }_{6}$ in vitro. Bacterially expressed and purified FLCN-His 6 was bound to Ni-NTA agarose and then incubated with 10 ng pure FLAG-FNIP1. FLCN-His 6 pulldown and FLAG-FNIP1 co-pulldown were assessed by immunoblotting. (f) FLCN-His, FLAG-FNIP1 and Hsp90 $\alpha$ tri-complex in vitro. Bacterially expressed and purified FLCN-His 6 was bound to Ni-NTA agarose and followed by incubation with 10 ng FLAG-FNIP1 and then 10 ng untagged Hsp90 $\alpha$ in vitro. FLCN-His 6 pulldown, and FLAG-FNIP1 and $\mathrm{Hsp} 90 \alpha$ co-pulldown were examined by immunoblotting.

affinity gel, we observed FNIP1 interaction with the Hsp90 $\alpha$ $\mathrm{M}$ - and C-domains, whereas FNIP2 interacted with only the M-domain of Hsp90 $\alpha$ (Fig. 3a). Association of FLCN with the Mand C-domains of Hsp90 $\alpha$ was also observed (Fig. 3a).

We next determined the region in FNIP1 that interacts with Hsp90. FNIP1 does not have a known functional domain; however, based on previous studies ${ }^{8,10}$, we constructed and transiently expressed four regions of HA-FNIP1 designated A-D in HEK293 cells (Fig. 3b). Consistent with the previous work ${ }^{8,10}$, truncation of FNIP1 at the C-terminus (deletion of fragment D) abrogated its interaction with FLCN (Fig. 3b). However, the concomitant deletion of 300 amino acids (fragment A) from the N-terminus of FNIP1 restored its binding to FLCN (Fig. 3b). This result is not in agreement with the previous study ${ }^{8}$ and underlies the complexity in FNIP1 binding to FLCN. Our data also indicated that the C-domain of FNIP1 (amino acid 929-1166 or fragment D) preferentially interacts with Hsp90 (Fig. 3b). We confirmed these data by carrying out a reciprocal experiment and co-expressing FNIP1-D-HA and Hsp90 $\alpha-$ FLAG full-length (wild type) and its different domains in HEK293 cells. We immunoprecipitated FNIP1-D-HA and observed co-IP of Hsp90 $\alpha$ full length and its M-domain (Fig. 3c). We next 
a

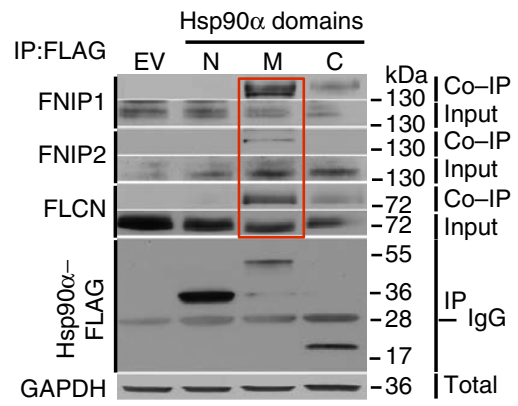

b

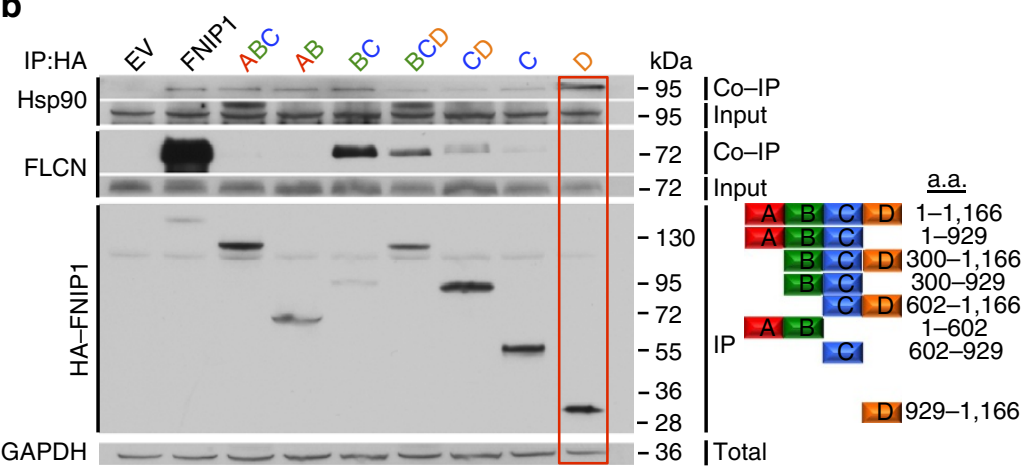

C

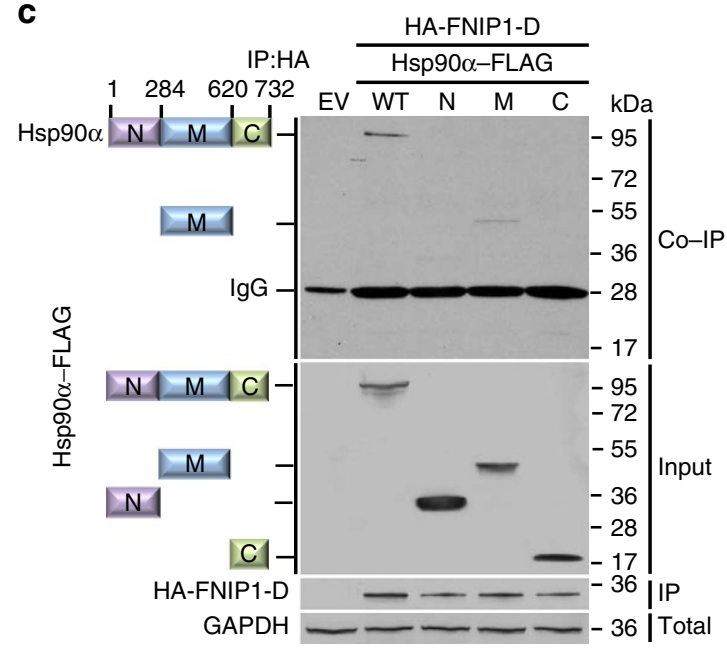

d

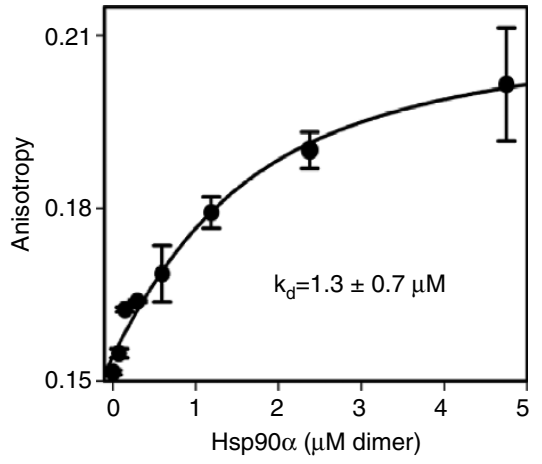

Figure 3 | FNIPs directly bind to Hsp90 M- and C-domains. (a) FLAG-tagged Hsp90 $\alpha-\mathrm{N}$-, M-, and C-domains were transiently expressed and immunoprecipitated from HEK293 cells. Co-IP of endogenous FLCN and FNIPs were assessed by immunoblotting. Empty vector (EV) was used as a control. (b) HA-tagged FNIP1 domains were transiently expressed and isolated from HEK293 cells. Co-IP of endogenous FLCN and Hsp90 were assessed by immunoblotting. Empty vector (EV) was used as a control. (c) FLAG-tagged Hsp90 $\alpha$ domains were transiently co-expressed with HA-tagged FNIP1-D in HEK293 cells. HA-FNIP1-D was immunoprecipitated and Hsp90 $\alpha$-FLAG co-IP was evaluated by immunoblotting. (d) Bacterially expressed and purified FNIP1-D and Hsp90 $\alpha$ binding affinity measured by fluorescence anisotropy. The error bars represent mean \pm s.d. of three independent experiments.

bacterially expressed and purified fragment D (FNIP1-D-His 6 ), (Supplementary Fig. 2b,c) and examined its affinity for binding to Hsp90 $\alpha$ in vitro by fluorescently labelling FNIP1-D-His ${ }_{6}$ with Texas Red Maleimide, and measured the $K_{\mathrm{d}}$ by fluorescence anisotropy (Fig. 2j). Our bacterially expressed and purified HSP90 $\alpha$ had ATPase activity. The titration fit to a single-site binding equation with a $K_{\mathrm{d}}$ of $1.3 \pm 0.7 \mu \mathrm{M}$ (Fig. $3 \mathrm{~d}$ ). This $K_{\mathrm{d}}$ was unaffected by the presence, absence or identity of adenosine nucleotide bound to the protein (Supplementary Fig. 2d). Taken together, our findings suggest that FNIPs behave as cochaperones of Hsp90 and they are involved in loading of FLCN to Hsp90.
FNIPs regulate Hsp90 activity and chaperoning of the clients. To gain further insight into FNIP1 and FNIP2 function as co-chaperones of Hsp90, we used small interfering RNA (siRNA) to silence either FNIP1 or FNIP2 (Supplementary Table 2), or both in HEK293 cells, and monitored the stability and activity of the Hsp90 kinase clients such as B-Raf, Ulk1 and Cdk4, and nonkinase clients, for example, GR, ER and FLCN (Fig. 4a and Supplementary Fig. 3a). Silencing either FNIP1 or FNIP2 caused a modest decrease in client protein levels. This data suggests that the absence of either FNIP1 or FNIP2 allows for compensation by the other FNIP isoform. Silencing of both FNIP1 and FNIP2 significantly decreased the stability of the selected kinase and 
a
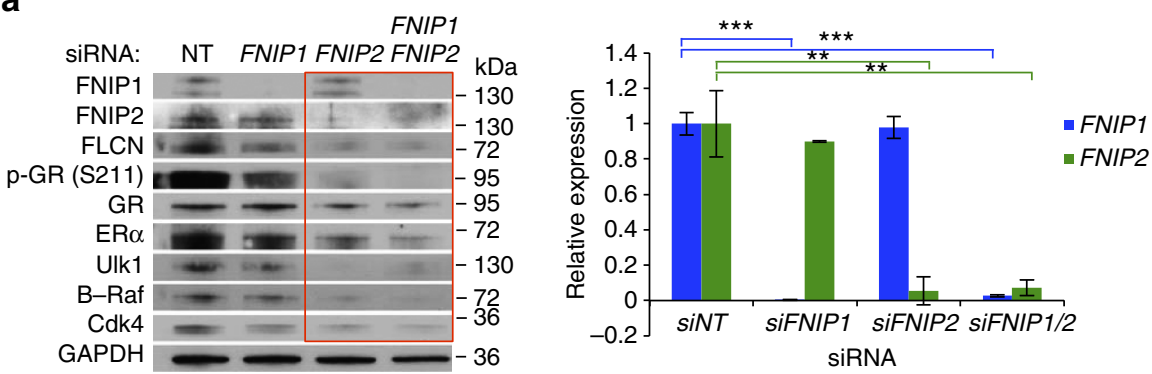

b

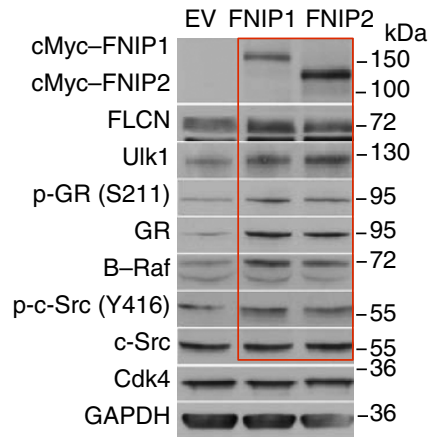

d

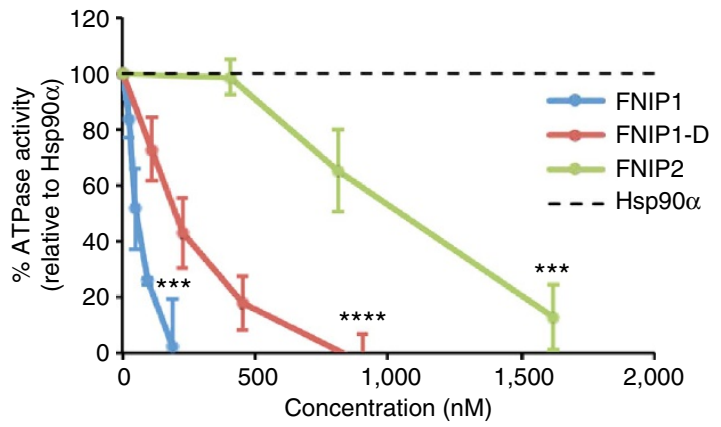

C
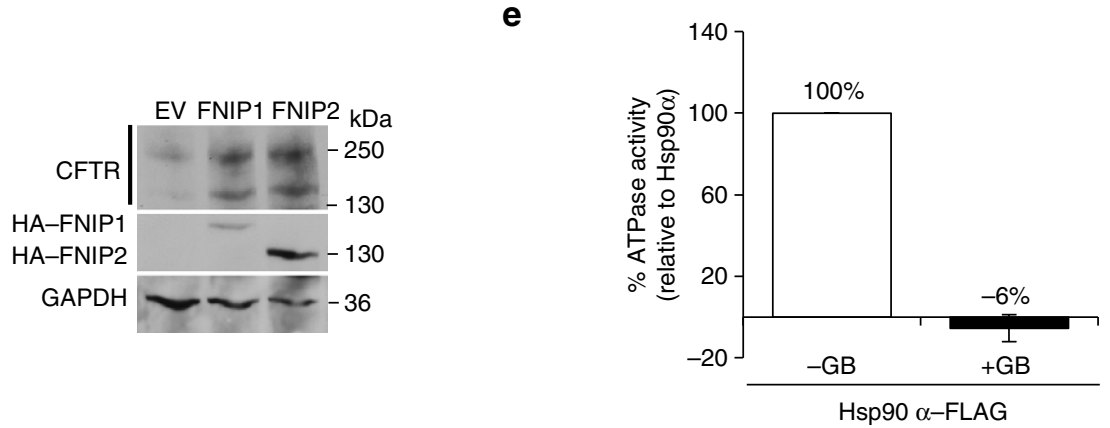

Figure 4 | FNIPs co-chaperones inhibit Hsp90 chaperone cycle and facilitate chaperoning of the clients. (a) Effect of siRNA knockdown of FNIP1 and FNIP2 on Hsp90 clients. Stability and activity of the indicated clients were assessed by immunoblotting. Densitometry of the western blotting for FNIP1 and FNIP2 is represented as mean \pm s.d. A Student's $t$-test was performed to assess statistical significance ( ${ }^{\star \star} P<0.005$ and $\left.{ }^{\star \star \star} P<0.0005\right)$. (b) Transient overexpression of cMyc-tagged FNIP1 or FNIP2 in HEK293 cells and their impact on levels of Hsp90 clients was assessed by immunoblotting. Empty vector (EV) was used as a control. (c) HEK293 cells were co-transfected with CFTR and indicated HA-FNIP1 and HA-FNIP2 constructs. After $24 \mathrm{~h}$, CFTR and FNIPs-HA were detected by immunoblotting; GAPDH was used as loading control. Empty vector (EV) was used as a control. (d) In vitro ATPase activity of Hsp90 $\alpha$-FLAG isolated from PC3 cells. Inhibitory effects of purified HA-FNIP1, FNIP1-D-HA or HA-FNIP2 on ATPase activity of Hsp90 $\alpha$-FLAG. All the data represent mean \pm s.d. A Student's $t$-test was performed to assess statistical significance $\left({ }^{\star \star \star} P<0.0005\right.$ and $\left.{ }^{\star \star \star \star} P<0.0001\right)$. (e) ATPase activity of Hsp90 $\alpha$-FLAG from d was inhibited by addition of $10 \mu \mathrm{M}$ GB. All the data represent mean \pm s.d. A Student's $t$-test was performed to assess statistical significance $\left({ }^{\star \star \star \star} P<0.0001\right)$.

non-kinase client proteins (Fig. 4a). We next transiently overexpressed cMyc-FNIP1 or cMyc-FNIP2 in HEK293 cells and examined the stability and the activity of the Hsp90 clients. Overexpression of FNIPs caused an increase in GR, Ulk1 and FLCN protein levels and hyperphosphorylation of B-Raf and pY416-c-Src (Fig. 4b and Supplementary Fig. 3b).

Cystic fibrosis transmembrane conductance regulator (CFTR) is also an Hsp90 client ${ }^{17,18}$ and relies on a 'slow' Hsp90 chaperone cycle for proper folding. To examine the effect of FNIPs on Hsp90 chaperone function, we assessed their impact on steady-state expression of CFTR protein in mammalian cells. HEK293 cells were transiently co-transfected with CFTR, and HA-FNIP1 and HA-FNIP2. Empty plasmid pcDNA3 (empty vector, EV) was used as a negative control. Western blot analysis of these samples using anti-CFTR antibody detected a doublet (Fig. 4c), with the upper band representing the mature Golgi- processed glycoform of CFTR found at the cell surface and the lower band an immature core-glycosylated protein (Fig. 4c). Overexpression of the FNIPs produced a significant increase in CFTR protein (Fig. 4c). These data suggested a reduction in Hsp90 chaperone activity and, therefore, an increase in CFTR expression.

Hsp90 chaperone function is coupled to its ATPase activity ${ }^{3}$. We therefore examined the impact of FNIPs on Hsp90 ATPase activity. We transiently expressed Hsp $90 \alpha-\mathrm{HA}$ in the prostate cancer PC3 cell line and HA-FNIP1, HA-FNIP1-D (amino acids 929-1166) and HA-FNIP2 in HEK293 cells. Proteins were immunoprecipitated, salt stripped and competed off the HA affinity beads with the relevant peptides (Supplementary Fig. 4a). The quality of the isolated proteins was examined by Coomassie staining of the SDS-polyacrylamide gel electrophoresis (SDSPAGE). These purified proteins were quantified and used in the 
a

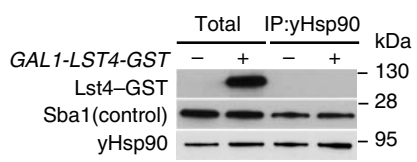

C

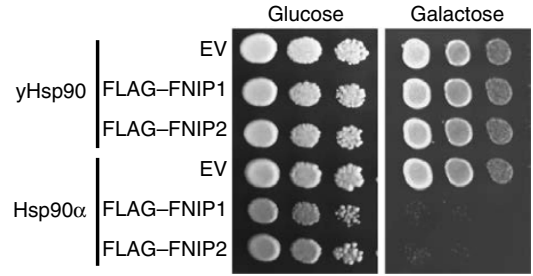

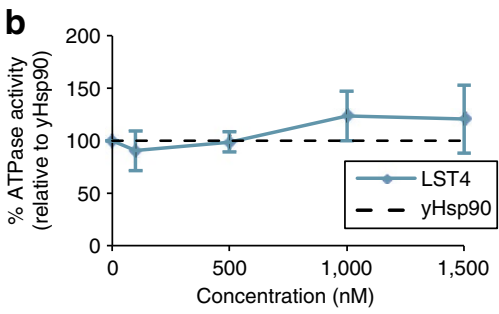

d

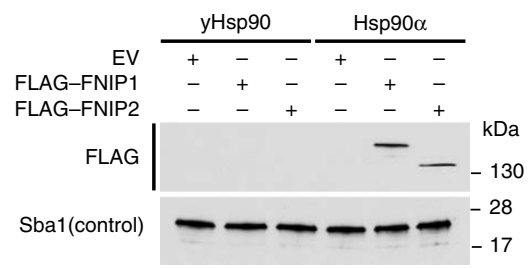

Figure 5 | Lst4 is not an orthologue of FNIPs in yeast. (a) BY4741 yeast strain expressing GAL1-LST4-GST was grown on either YPED (glucose) or YPGal (Galactose) media at $30^{\circ} \mathrm{C}$ for $8 \mathrm{~h}$. Endogenous yHsp90 immunoprecipitation (IP) and co-IP Lst4-GST was detected by immunoblotting. (b) In vitro ATPase

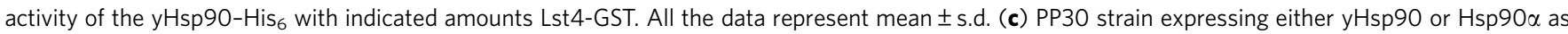
the sole copies of Hsp90 were transformed by GAL1-FLAG-FNIP1 or GAL1-FLAG-FNIP2. Cells were spotted at 1:10 dilution series on YPED or YPGal media. Plates were incubated at $30^{\circ} \mathrm{C}$ for 2 days. (d) Yeast strains in $\mathbf{i}$ were grown on YPGal liquid media for $12 \mathrm{~h}$ and the expression of FLAG-FNIP1 and FLAG-FNIP2 were examined by immunoblotting.

molar ratio indicated in Supplementary Fig. 4b of Hsp90 $\alpha$ :FNIP in the PiPer Phosphate Assay Kit (Thermo Fisher Scientific), in the presence of ATP as substrate as previously described (see Methods $)^{19}$. We measured the ATPase activity of isolated Hsp $90 \alpha$ in vitro as previously described ${ }^{19}$ (Fig. $4 \mathrm{~d}$ and Supplementary Fig. 4c). GB $(10 \mu \mathrm{M})$ inhibited ATPase activity (Fig. 4e). Addition of FNIP1, FNIP1-D and FNIP2 also significantly inhibited the ATPase activity of Hsp90 $\alpha$ (Fig. 4d and Supplementary Fig. 4c). Percentage ATPase activity was based on $\mathrm{mmol} P_{\mathrm{i}}$ per mol min ${ }^{-1}$ for Hsp90 $\alpha$ alone and in the presence of FNIPs titrated until inhibition was achieved $($ Hsp $90 \alpha=291.5 \mathrm{nM}, \mathrm{FNIP} 1=189.8 \mathrm{nM}$, FNIP1-D $=899.3 \mathrm{nM}$ and FNIP2 $=1.6 \mu \mathrm{M}$ ) (Supplementary Fig. 4d). These data suggest that FNIPs are potent inhibitors of the Hsp90 chaperone cycle.

Hsp90 is an evolutionarily conserved chaperone and based on structural homology detection methods Lst4 is a potential orthologue of FNIPs in Saccharomyces cerevisiae ${ }^{20}$. However, we were unable to detect Lst4-GST interaction with yeast Hsp90 (yHsp90, also known as Hsp82) (Fig. 5a). In addition, unlike FNIPs, Lst4-GST was unable to inhibit ATPase activity of yHsp90 in vitro (Fig. $5 \mathrm{~b}$ and Supplementary Fig. $4 \mathrm{e}, \mathrm{f}$ ). We next decided to express FLAG-FNIP1 and FLAG-FNIP2 galactose-inducible promoter of GAL1 in yeast containing either human or yeast Hsp90 as the sole functional Hsp90. Overexpression of FNIPs in yeast containing human Hsp90 $\alpha$ caused lethality (Fig. 5c). However, overexpression of FNIPs in yeast with only yHsp90 did not cause any growth defects. We examined the expression of the FNIPs in these cells by western blot analysis and were only able to detect FLAG-FNIP1 and FLAG-FNIP2 in yeast expressing Hsp90 $\alpha$ (Fig. 5d). These data suggest that expression of FNIPs in yeast cause lethality, because they inhibit the essential chaperone function of Hsp90 $\alpha$. Taken together, our results suggest that (i) Lst4 is not an orthologue of FNIPs in yeast and (ii) FNIPs probably arose in higher eukaryotes as co-chaperones to inhibit or slow the complex chaperone cycle of Hsp90 in cellular milieu.

FNIPs compete with the Ahal co-chaperone for binding to Hsp90. To determine the effects of FNIPs on Hsp90 interaction with co-chaperones, we used FNIP1- and FNIP2-specific siRNA to silence expression of these genes in HEK293 cells transiently expressing Hsp90 $\alpha$-FLAG. We next immunoprecipitated Hsp90 $\alpha$-FLAG from these cells and examined its interaction with selected co-chaperones. Our data show that siRNA-mediated silencing of both FNIP1 and FNIP2 increased Hsp90 interaction with the co-chaperones Ahal and PP5 (Fig. 6a). We next transiently overexpressed HA-FNIP1 and HA-FNIP2 in HEK293 cells that also express Hsp90 $\alpha$-FLAG. The overexpression of FNIPs compared with the endogenous FNIP1 and FNIP2 were examined by immunoblotting (Supplementary Fig. 5a). Following IP of Hsp $90 \alpha-F L A G$, we observed a marked reduction in its interaction with the co-chaperones Ahal and PP5 (Fig. 6b). Aha1 is the activator of the Hsp90 ATPase activity ${ }^{5,6}$. We next queried whether Ahal has the ability to stimulate the ATPase activity of Hsp90 that has already bound to FNIP1, FNIP1-D or FNIP2. In agreement with our earlier data, FNIPs inhibited the ATPase activity of Hsp90 $\alpha$ and, conversely, addition of $1.3 \mu \mathrm{M}$ Aha1 to these reactions stimulated Hsp90 ATPase activity (Fig. $6 c$ and Supplementary Fig. 5b-f). Taken together, these data suggest that Ahal has the ability to displace FNIPs from Hsp90. We obtained additional evidence for this possibility by using the bacterially expressed and purified Hsp90 $\alpha$, Aha1-FLAG and FNIP1-D fragment. First, we bound FNIP1-D-His 6 to Ni-NTA agarose and incubated with $100 \mathrm{ng}$ recombinant Hsp90 $\alpha$. After washing the agarose with buffer, we added different amounts of Aha1-FLAG. Our data show that addition of $10 \mathrm{ng}$ recombinant Aha1-FLAG completely dissociated Hsp90 $\alpha$ from FNIP1-D-His 6 (Fig. 6d). We next carried out a similar experiment by first immobilizing Aha1-FLAG onto anti-FLAG M2 affinity gel. We then added $100 \mathrm{ng}$ recombinant Hsp90 $\alpha$, to form an Aha1-Hsp90 $\alpha$ complex. After washing the agarose beads with buffer, we added different amounts of FNIP1-D-His ${ }_{6}$. We observed that addition of $100 \mathrm{ng}$

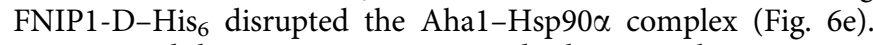
We repeated the experiments in Fig. 6d,e, but using bovine serum albumin as a negative control (Supplementary Fig. 5g-i). Our data confirm that Ahal and FNIP1 compete for binding to Hsp90, either accelerating or decelerating its chaperone cycle. In addition, Ahal appears to have higher affinity than FNIP1 
a
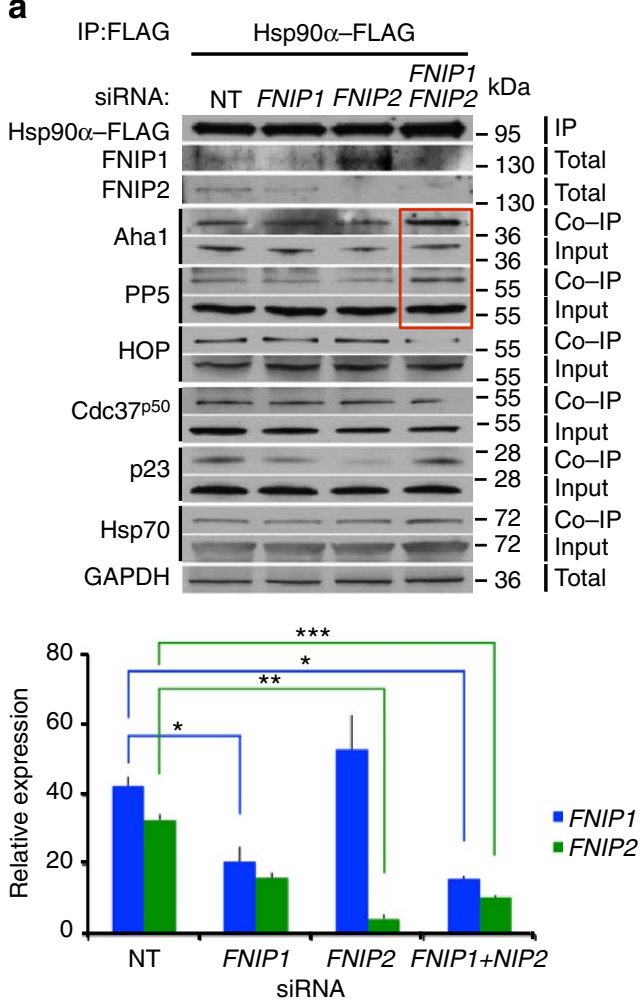

b

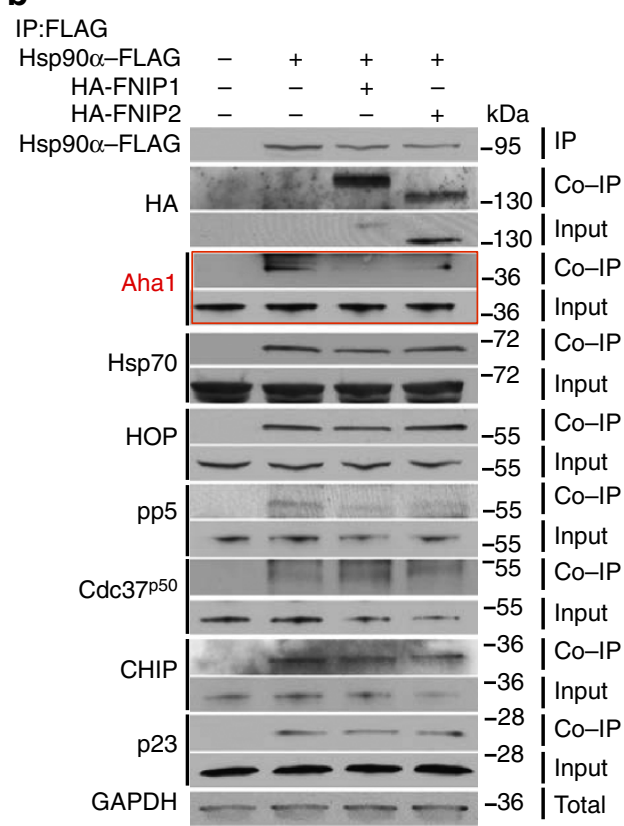

c

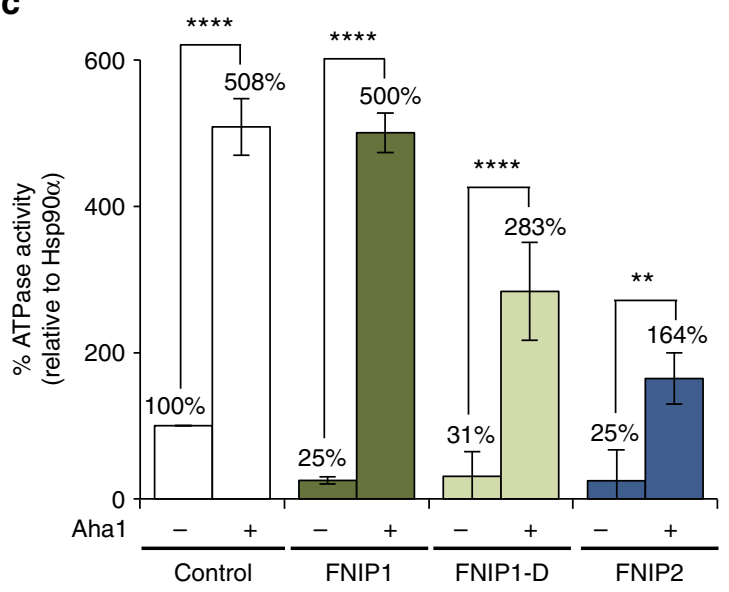

\begin{tabular}{|lr|}
\hline Comparison & \multicolumn{1}{c|}{\begin{tabular}{c}
-value \\
\hline Hsp90 vs Hsp90+Aha1
\end{tabular}} \\
\hline Hsp90+FNIP1 vs Hsp90+FNIP1+Aha1 & $<0.0001$ \\
\hline $\begin{array}{l}\text { Hsp90+FNIP1-D vs Hsp90+FNIP1-D } \\
\text { +Aha1 }\end{array}$ & $<0.0001$ \\
\hline Hsp90+FNIP2 vs Hsp90+FNIP2+Aha1 & $<0.005$ \\
\hline
\end{tabular}

d

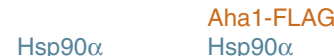

IP: FNIP1-D-His $\quad$ IP: FNIP1-D-His $\quad$ IP: FNIP1-D-His

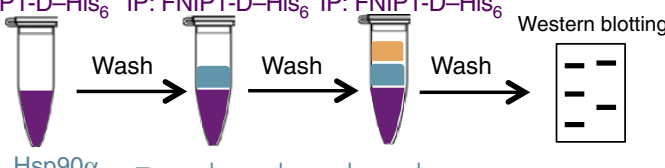

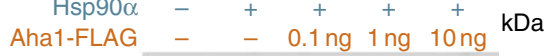

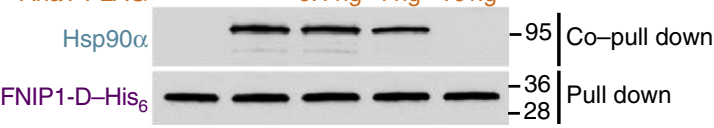

e

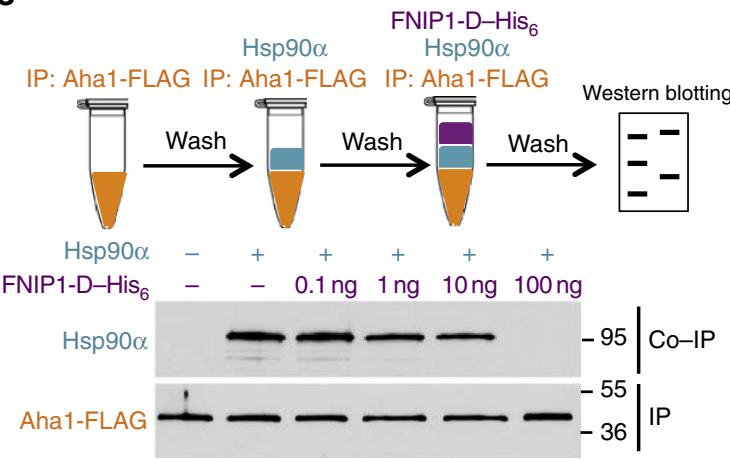

Figure 6 | FNIPs compete with the Aha1 co-chaperone for binding to Hsp90. (a) Hsp90 $\alpha$-FLAG was transiently expressed in HEK293 cells for $24 \mathrm{~h}$ followed by siRNA knockdown of FNIP1 and/or FNIP2. Hsp90 $\alpha$-FLAG was immunoprecipitated (IP) and co-IP of the co-chaperones was assessed by immunoblotting. Densitometry of the western blotting for FNIPs is represented as mean \pm s.d. A Student's $t$-test was performed to assess statistical significance $\left({ }^{\star} P<0.05,{ }^{\star \star} P<0.005\right.$ and $\left.{ }^{\star \star \star} P<0.0005\right)$. (b) HEK293 cells were transiently co-transfected with Hsp90 $\alpha-F L A G$ and HA-FNIP1 or HA-FNIP2. $\mathrm{Hsp} 90 \alpha$-FLAG was isolated and co-IP of co-chaperones examined by immunoblotting. (c) HA-FNIP1, HA-FNIP1-D and HA-FNIP2 inhibited Hsp90 $\alpha-H A$ ATPase activity after $30 \mathrm{~min}$. Addition of $1.3 \mu \mathrm{M}$ Aha1-FLAG stimulated the ATPase activity. All the data represent mean \pm s.d. A Student's $t$-test was performed to assess statistical significance ${ }^{\star \star} P<0.005$ and $\left.{ }^{\star \star \star \star} P<0.0001\right)$. (d) FNIP1 and Aha1 compete for binding to Hsp90 $\alpha$. FNIP1-D-His 6 was attached to Ni-NTA agarose and then incubated with $\mathrm{Hsp} 90 \alpha$. Ni-NTA agarose was then washed and incubated with the indicated amounts of Aha1-FLAG. (e) Aha1-FLAG attached to anti-FLAG M2 affinity gel was incubated with Hsp90 $\alpha$ initially and then washed and incubated with indicated amounts of the FNIP1-D-His 6 . 
a

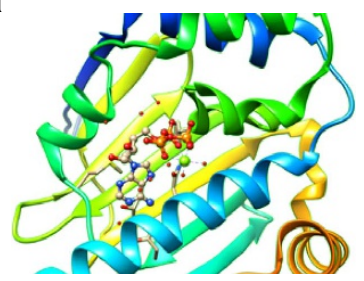

C

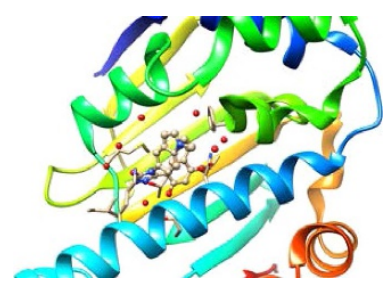

b

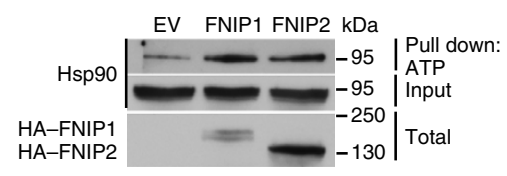

d

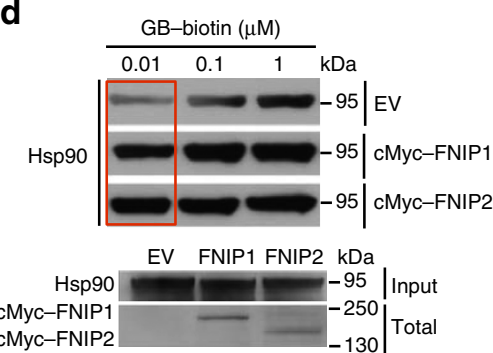

f
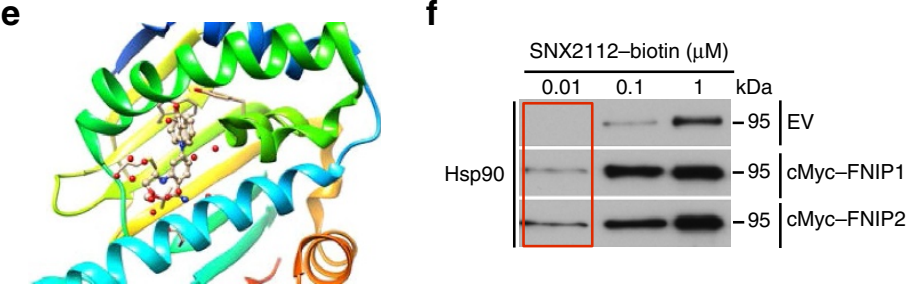

g

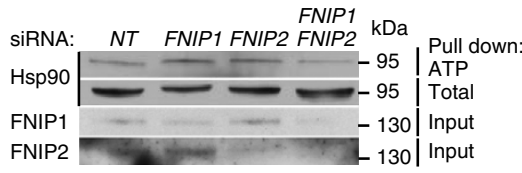

h



Figure 7 | Overexpression of FNIPs enhance Hsp90 binding to drugs. (a) Structure of human Hsp90 $\alpha$ N-domain bound to ATP (PDB: 3TOZ). (b) HA-FNIP1, HA-FNIP2 or empty vector (EV) were transiently overexpressed in HEK293 cells. Lysates were incubated with ATP agarose. Hsp90 binding to ATP agarose was examined by immunoblotting. (c) Structure of human Hsp90 $\alpha$ N-domain bound to GB (PDB: 3TUH). (d) cMyc-FNIP1, cMyc-FNIP2 or empty vector (EV) were transiently overexpressed in HEK293 cells. Lysates were incubated with indicated amounts of biotinylated GB followed by streptavidin agarose beads. Hsp90 was detected by immunoblotting. (e) Structure of human Hsp90 $\alpha$ bound to related SNX2112 compound, tetrahydro-4Hcarbazol-4-one (PDB: 3DOB). (f) Lysates from $\mathbf{d}$ were incubated with indicated amounts of biotinylated SNX2112 followed by streptavidin agarose beads. Hsp90 was detected by immunoblotting. (g) FNIP1 and FNIP2 were silenced by siRNA in HEK293 cells. Lysates were incubated with ATP agarose. Hsp90 binding to ATP agarose was examined by immunoblotting. NT represents non-targeting siRNA control pools. (h) Lysates from $\mathbf{g}$ were incubated with indicated amounts of biotinylated GB followed by streptavidin agarose beads. Hsp90 was detected by immunoblotting.

towards Hsp90, as $10 \mathrm{ng}$ of Aha1 was sufficient to displace FNIP1-D from Hsp90 (Fig. 6d).

FNIPs overexpression enhances Hsp90 binding to ATP and drugs. ATP binding and hydrolysis by the N-terminus of Hsp90 is essential for its chaperone function (Fig. 7a) ${ }^{3}$. We examined the impact of FNIPs on Hsp90 binding to ATP or drugs by first overexpressing HA-FNIP1 and HA-FNIP2 in HEK293 cells. Overexpression of FNIPs caused an increase in Hsp90 binding to ATP agarose (Fig. 7b). Furthermore, overexpression of cMycFNIP1 and cMyc-FNIP2 in HEK293 cells significantly increased the Hsp90 binding to $0.01 \mu \mathrm{M}$ biotinylated-GB (Fig. 7c,d) and $0.01 \mu \mathrm{M}$ biotinylated-SNX2112 (ref. 14 and Fig. 7e,f) compared with control (EV). We next examined the effects of FNIP1 and FNIP2 knockdown on Hsp90 binding to ATP and drug. siRNA knockdown of FNIP1, FNIP2 or both in HEK293 cells did not have an impact on Hsp90 binding to ATP (Fig. 7g) but significantly decreased Hsp90 binding to GB (Fig. 7h).

We next confirmed that overexpression of either FNIP1-HA or FNIP2-HA (Fig. 8a,b) sensitized the HEK293 cells to GB, as evidenced by induction of the pro-apoptotic markers cleaved caspase-3 and cleaved poly-ADP-ribose polymerase (PARP) (Fig. 8c). Conversely, siRNA knockdown of FNIP1 or FNIP2 in HEK293 cells significantly reduced apoptosis with $1 \mu \mathrm{M}$ GB and knockdown of both FNIPs completely abolished the induction of apoptotic markers (Fig. 8d). Taken together, these data suggest that expression of FNIPs correlates to Hsp90 binding to drugs and also sensitivity of cells to Hsp90 inhibitors.

FNIPs overexpression sensitizes cancer cells to Hsp90 drugs. Tumours are generally sensitive to Hsp90 inhibitors $19,21,22$. We therefore asked whether FNIPs are overexpressed in cancer cells and also whether they are contributing to cancer cell sensitivity towards Hsp90 inhibitors. We used prostate (LNCaP), bladder (T24), breast (MCF7), lung (H1299) and colorectal adenocarcinoma (HT29) cancer cell lines. Previous work by Synta Pharmaceuticals Corp. (data available at www.syntapharma.com/) has established these cell lines' sensitivity to Hsp90 inhibitor GB and these are: $\mathrm{LNCaP}(\mathrm{IC} 50=8 \mathrm{nM}), \mathrm{T} 24(\mathrm{IC} 50=7 \mathrm{nM})$, MCF7 $(\mathrm{IC} 50=25 \mathrm{nM}), \mathrm{H} 1299 \quad(\mathrm{IC} 50=6 \mathrm{nM})$ and HT29 $(\mathrm{IC} 50=50 \mathrm{nM})$. We first showed that FNIPs were highly expressed in these cancer cells compared with HEK293 cells (Fig. 9a). We next immunoprecipitated the endogenous Hsp90 from the above cancer cells and detected its interaction with FNIPs (Fig. 9a). To link the high levels of FNIPs with sensitivity of cancer cells to Hsp90 inhibitors, we used siRNA to knock down FNIP1 and FNIP2 in LNCaP, T24, MCF7, H1299 and HT29 cells. The apoptotic markers cleaved caspase- 3 and cleaved PARP were abundant in cancer cells treated with $0.1 \mu \mathrm{M}$ 
a



b

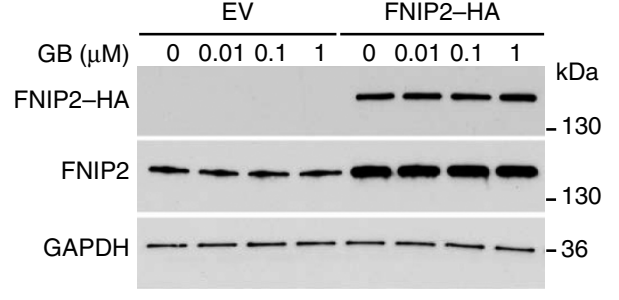

C

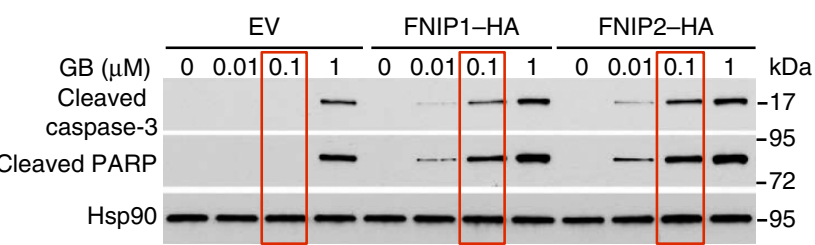

d

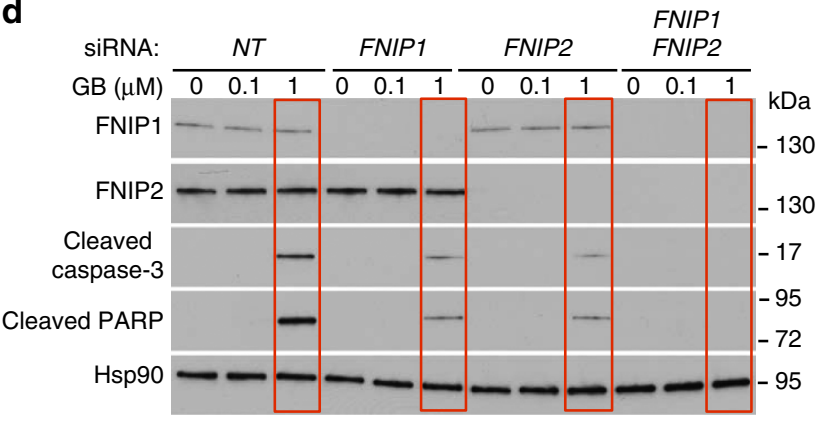

Figure 8 | Overexpression of FNIPs sensitize HEK293 cells to Hsp90

inhibitor. (a) HEK293 cells were transiently transfected with HA-FNIP1 or empty vector (EV), (b) or HA-FNIP2 or empty vector (EV), and treated with indicated amounts of GB. (c) Induction of apoptotic markers in lysates from $\mathbf{a}$ and $\mathbf{b}$ were shown by immunoblotting using anti-cleaved caspase-3 and cleaved PARP antibodies. (d) FNIP1, FNIP2 or both were silenced by siRNA in HEK293 cells and then treated with indicated amounts of GB. Apoptotic markers were examined by immunoblotting using anti-cleaved caspase-3 and cleaved PARP antibodies.

GB; however, this effect was abrogated in the siRNA FNIPs knockdown samples (Fig. 9b-f). These data suggest that overexpression of FNIPs is a contributing factor towards cancer cell sensitivity to Hsp90 inhibitor GB. It is noteworthy that cleaved caspase-7 was used to evaluate apoptosis in MCF7 cells, as they lack caspase-3 (Fig. 9d).

Elevated FNIPs sensitize renal tumours to Hsp90 inhibitor. To gain further insight into FNIPs expression and sensitivity of tumours to Hsp90 inhibitors, we examined tumours and adjacent normal tissues from patients with renal cell carcinoma (RCC), the most common type of kidney cancer ${ }^{23}$. Histopathologically, RCCs are classified into subtypes. We used tumours from patients with clear cell RCC, papillary type I and type II RCC, and oncocytoma (Fig. 10a-d). Within 10 min of removal of tumours, by radical or partial nephrectomy, RCC tumours and adjacent normal tissues were dissected into $3 \mathrm{~mm}^{3}$ pieces followed by protein extraction and drug binding assay. Our data showed that both FNIP1 and FNIP2 were overexpressed in RCC tumours compared with adjacent normal tissues (Fig. 10a-d). In addition, we discovered that the Hsp90 from RCC tumours had a higher affinity for binding to biotinylated GB compared with the adjacent normal tissues (Fig. 10a-d). We next showed greater association between Hsp90 and FNIPs compared with normal tissues (Fig. 10e). This is not surprising, because FNIPs are highly expressed in tumours compared with adjacent normal tissues. However, we found that Hsp90 bound stronger to Ahal in normal tissues compared with the tumours, even though both tissues expressed equal levels of Ahal (Fig. 10e). Our earlier data showed that FNIPs compete with the activating co-chaperone Aha1 for binding to Hsp90 (Fig. 6e). We therefore asked whether addition of purified FNIP1-D-His 6 to the protein lysates from normal tissues could disrupt the Hsp90-Ahal complex. Addition of $100 \mathrm{ng}$ FNIP1-D-His 6 to protein lysates from normal tissues displaced Ahal interaction with Hsp90 and also increased Hsp90 binding to $0.1 \mu \mathrm{M}$ biotinylated GB (Fig. 10f). Taken together, our data suggest that FNIPs make renal tumours sensitive to Hsp90 inhibitors. Their expression level can potentially serve as a predictive indicator of tumour response to Hsp90 inhibitors.

\section{Discussion}

Germline mutation in the tumour suppressor FLCN is responsible for Birt-Hogg-Dubé syndrome, an inherited kidney cancer syndrome ${ }^{24}$. Previous work has shown that FNIP1 and FNIP2 are critical components of the FLCN complex and are essential for its tumour suppressive function ${ }^{9}$. However, the exact molecular function of FNIP1 and FNIP2 remains elusive. In this study, we demonstrate by MS analysis that FLCN interacts with the molecular chaperones Hsp70 and Hsp90, as well as members of the chaperonin system TRiC. Our data have shown that FLCN is a bona fide Hsp70 and Hsp90 client, as treating the cells with the Hsp70 inhibitor JG-98 destabilizes FLCN, and furthermore Hsp90 inhibitor treatment GB, SNX2112 or PU-H71 leads to dissociation of FLCN from Hsp90 and consequently its ubiquitination and proteasomal degradation. Based on our current knowledge in the function of TRiC-Hsp70-Hsp90 proteins and also our findings here, we would like to suggest that TRiC-Hsp70 are involved in 'early' stages of FLCN folding and Hsp90 plays a key role in protecting FLCN from degradation. We further demonstrated that FLCN binding partners FNIP1 and FNIP2 function as co-chaperones of Hsp90. FNIP1 interacts with the M- and C-domains of Hsp90, whereas FNIP2 interacts only with its M- domain. Previous work reported that FNIP1 and FNIP2 exist as a homodimer and a heterodimer, respectively ${ }^{10}$. We confirmed these data by demonstrating the existence of a FNIP1:FNIP2 heterodimer and its interaction with FLCN. We have also demonstrated that three populations of FNIP1 and FNIP2 interact with Hsp90; however, the FNIP1 homodimer preferentially binds to Hsp90. Our in vitro data provide additional evidence that recombinant FNIP1 directly interacts with Hsp90; however, FLCN requires the presence of FNIP1 for its binding to Hsp90. These data suggest that FNIP1 and FNIP2, similar to the co-chaperone Cdc37, are involved in 'loading' of FLCN and perhaps other client proteins to Hsp90. Approximately 93\% of all pathogenic FLCN mutations prematurely truncate the encoded FLCN protein ${ }^{25}$. When experimentally modelled in vitro, most mutations analysed appeared to be pathogenic by disrupting stability of FLCN ${ }^{25}$. In addition, FLCN binding to both FNIP1 and FNIP2 is mediated specifically through the C-terminal 
a
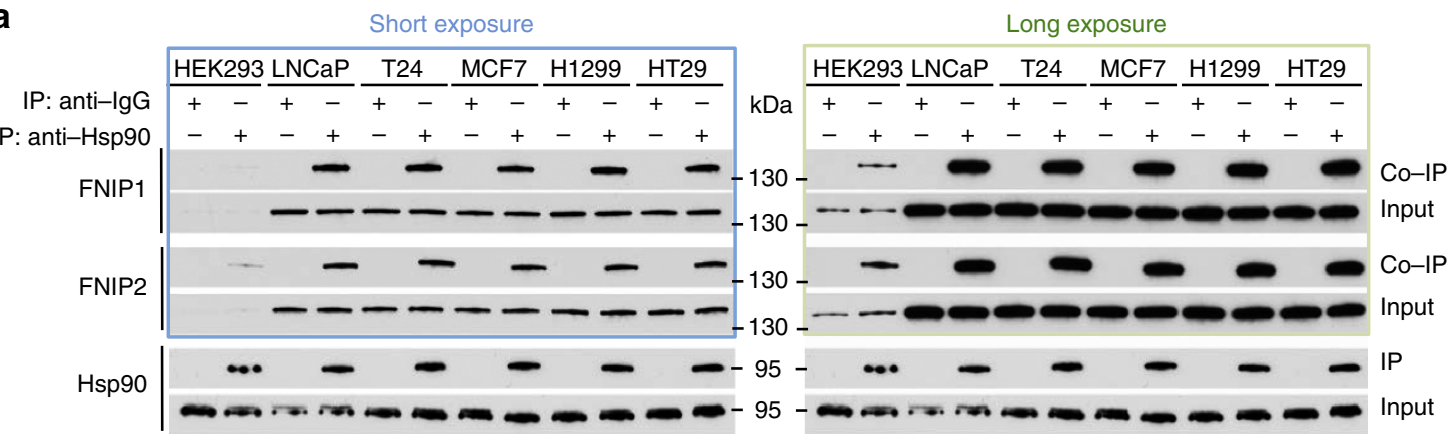

b

C

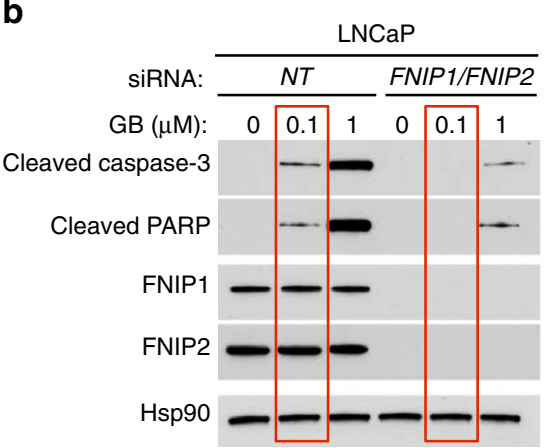

d
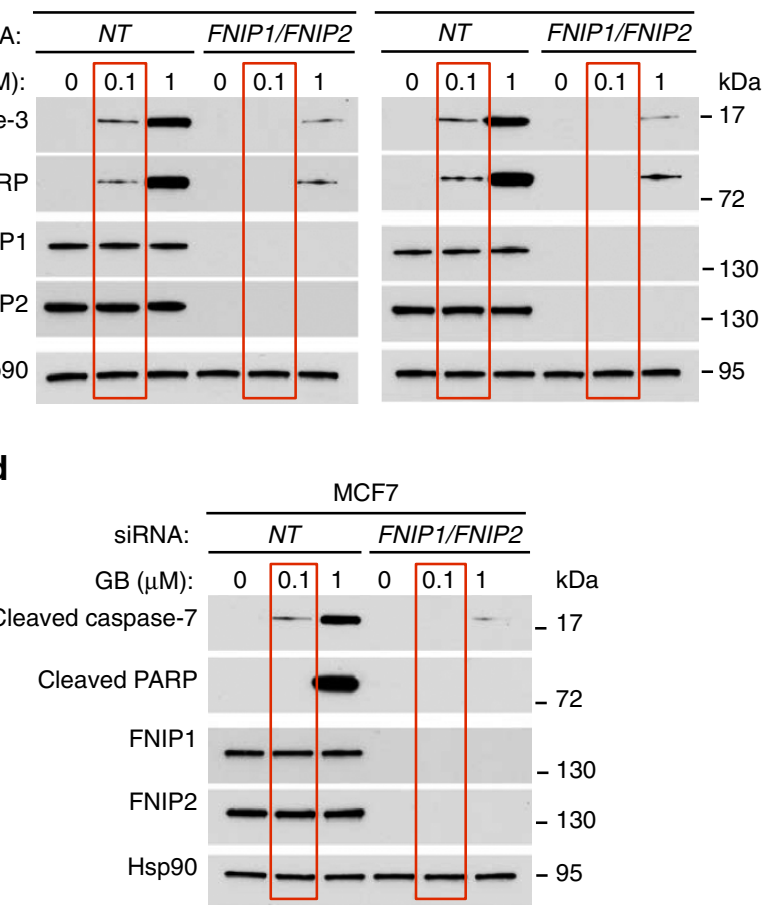

e

$\mathbf{f}$

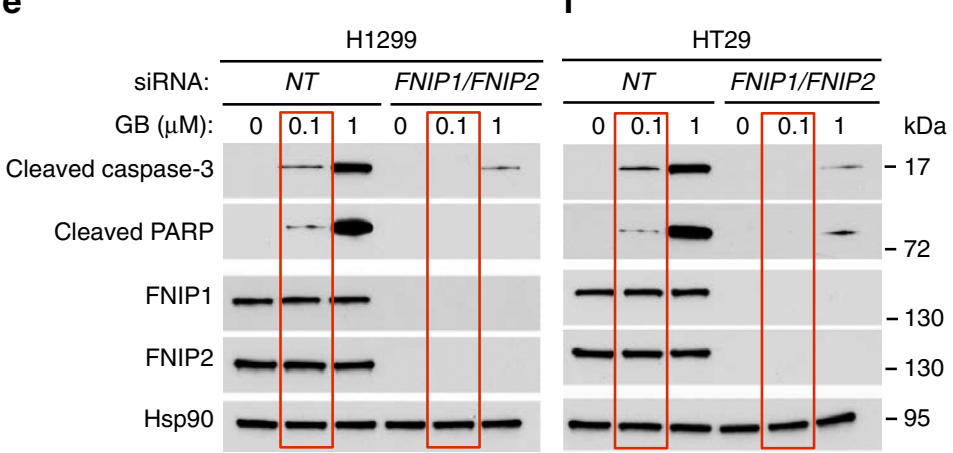

Figure 9 | FNIPs expression sensitizes cancer cells to Hsp90 inhibitor. (a) Hsp90 was immunoprecipitated from HEK293 cells and LNCaP (prostate), T24 (bladder), MCF7 (breast), H1299 (lung) and HT29 (colorectal) cancer cell lysates using anti-Hsp90 or lgG (control), and immunoblotted with FNIP1 and FNIP2 antibodies to confirm protein interaction. Short and long exposures of the blots are shown. (b) Both FNIP1 and FNIP2 were silenced by siRNA in LNCaP, (c) T24, (d) MCF7, (e) H1299 and (f) HT29, and then treated with indicated amounts of GB. Apoptotic markers were shown by immunoblotting using anti-cleaved caspase-3 and cleaved PARP antibodies. Anti-cleaved caspase-7 antibody was only used for MCF7 cells.

region of $\mathrm{FLCN}^{8,26}$. Truncating mutations result in loss of the C-terminus of FLCN, abolishing its interaction with FNIP1 and FNIP2, and consequently Hsp90. We speculate that loss of Hsp90 interaction with FLCN is the reason for the instability of the pathogenic FLCN mutants.
Hsp90 chaperone activity is coupled to its ATPase activity, which is tightly regulated by co-chaperones and posttranslational modification $^{27,28}$. Our results suggest that FNIP1 is a potent inhibitor of Hsp90 ATPase activity, as $200 \mathrm{nM}$ of FNIP1 inhibits Hsp90 ATPase activity by 50-fold. FNIP2 also has shown 
a
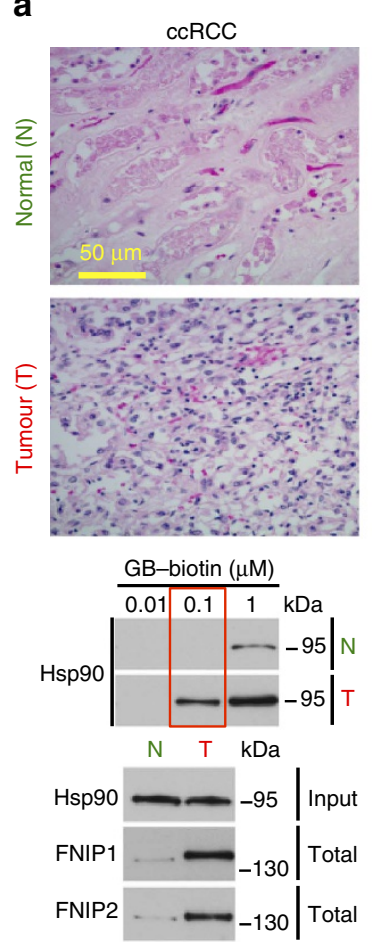

b
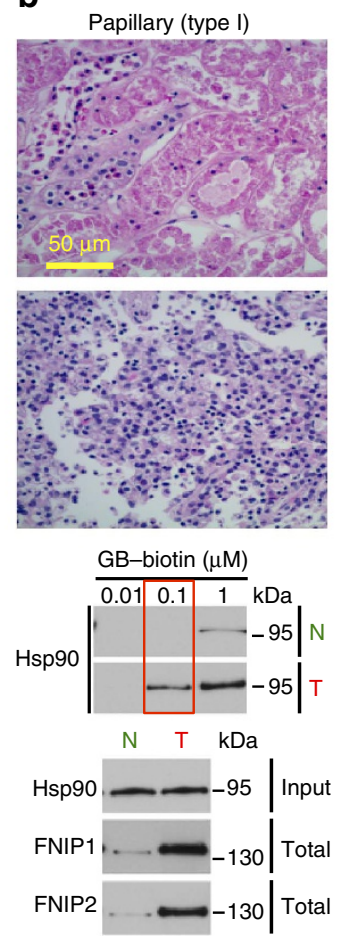

C
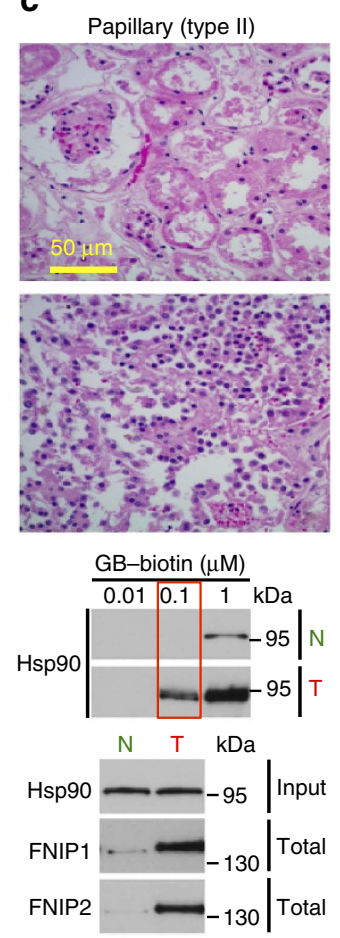

d
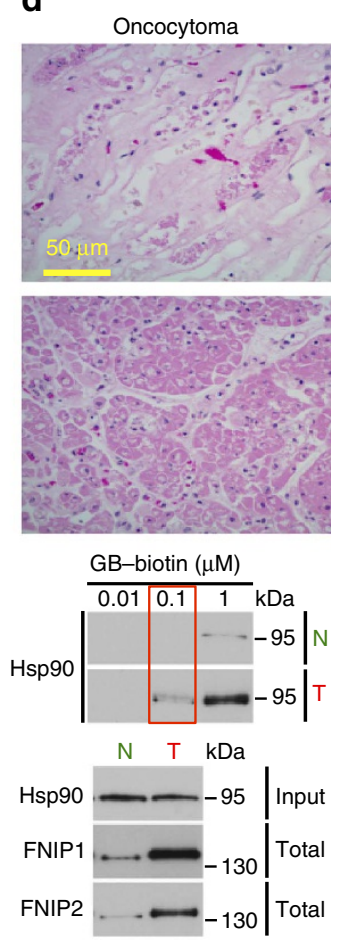

e

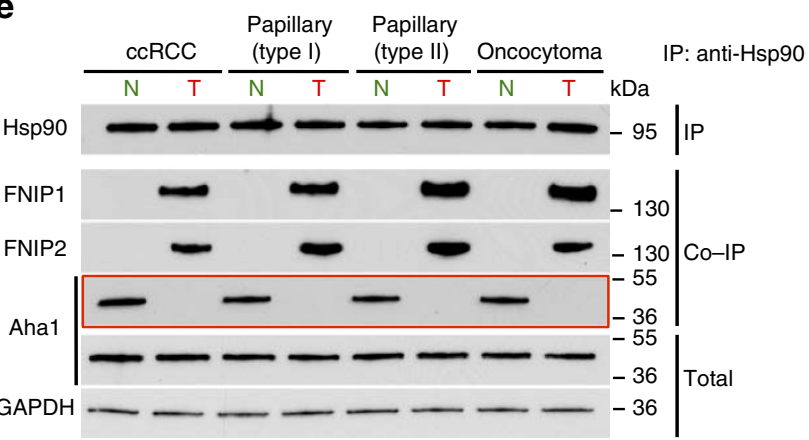

f



Figure 10 | High levels of FNIPs make renal tumours sensitive to Hsp90 inhibitor GB. (a) Clear cell renal cell carcinoma (ccRCC), (b) Papillary type I, (c) Papillary type II, (d) Oncocytoma (Tumours, T) and adjacent normal tissues (Normal, N) were stained with haematoxylin and eosin (H\&E). Proteins were also extracted from these tumours and adjacent normal tissues and incubated with indicated amounts of biotinylated GB followed by streptavidin agarose beads. Hsp90 was detected by immunoblotting. Expression of FNIP1 and FNIP2 in these samples was also detected by immunoblotting. (e) Hsp90 immunoprecipitated from tumours $(\mathrm{T})$ and adjacent normal tissues $(\mathrm{N})$ in a-d. Co-IP of FNIPs and Aha1 was examined by immunoblotting. (f) Lysates from normal tissues in a-d were incubated with or without $100 \mathrm{ng}$ of pure FNIP1-D-His ${ }_{6}$ for 10 min. Hsp90 was immunoprecipitated and co-IP of FNIP1-D-His 6 and Aha1 were shown by immunoblotting. 
inhibitory activity towards Hsp90; however, it required $1.6 \mu \mathrm{M}$ of FNIP2 to inhibit the ATPase activity by eightfold. Although we use the term 'inhibition' here, FNIPs seem only to be slowing the chaperone cycle. Their effect on Hsp90 is not similar to the small molecule inhibitors of Hsp90 that bind to the $\mathrm{N}$-domain and inhibit Hsp90 chaperone function. The reason for this conclusion stems from our observation that overexpression of FNIP1 or FNIP2 does not lead to degradation of Hsp90 client proteins. In addition, chaperoning of the proteins such as CFTR that require a slow chaperone cycle was enhanced by overexpression of FNIP1 and FNIP2. We have also shown that overexpression of FNIPs enhance Hsp90 binding to GB and SNX2112. Moreover, Hsp90 from kidney tumours bound stronger to GB compared with Hsp90 from adjacent normal tissues. This observation correlated with the overexpression of both FNIP1 and FNIP2 in tumours compared with adjacent normal tissues. Our findings suggest that FNIPs expression level can potentially serve as a predictive indicator of tumour response to Hsp90 inhibitors.

What is the relationship between FNIPs and Ahal towards binding to Hsp90? FNIPs bind to the M-domain of Hsp90 and may interfere with the $\mathrm{N}$-domain interaction with the catalytic domain. We also found that FNIPs compete with Ahal cochaperone binding to Hsp90. Our data have demonstrated the ability of FNIP1 and Ahal to compete for binding to Hsp90, fine tuning its chaperone cycle. The competition between Ahal and FNIPs binding to Hsp90 was also observed in renal tumours and their adjacent normal tissues. We found that Ahal bound stronger to Hsp90 in normal tissues; however, overexpression of FNIPs appears to displace Ahal from Hsp90 and increase Hsp90 binding to its inhibitors. Our data are in agreement with previously published work by Workman's laboratory ${ }^{29}$, suggesting that Aha1 dissociation from Hsp90 plays a role in drug sensitivity. What determines the binding of these proteins to Hsp90? We have already shown that posttranslational modifications of Hsp90 determine its binding to cochaperones ${ }^{28}$. In addition, our recent work has shown that c-Abl-mediated phosphorylation of Y223-Ahal promotes its interaction with Hsp90 (ref. 19). We therefore suspect similar mechanisms exist between these three proteins, where cross-talk between posttranslational modifications is a determining factor for differential binding of Ahal and FNIPs to Hsp90.

\section{Methods}

Plasmids and yeast strains. FLCN complementary DNA was synthesized in FLAG-tagged pcDNA3.1 vector backbone (Thermo Fisher Scientific) by GeneWiz, Inc. FNIP1-His 6 and FNIP2- His $_{6}$ messenger RNA was also synthesized in pcDNA3.1 by GeneWiz, Inc. and subcloned into pCMV-Myc and pCMVHA vectors (ClonTech). The EVs containing these tags were used and also referred to as controls. For bacterial expression of FNIP1, FNIP2, FNIP1-D and Hsp $90 \alpha$, pRSET-A expression vector containing a $6 \times$ His tag (ThermoFisher Scientific) was used. The HA-FNIP1 domain-specific plasmids were previously reported $^{8}$. Hsp90 $\alpha$ domain-specific plasmids were amplified using primers listed in Supplementary Table 3. FNIP1-FLAG and FNIP2-FLAG were cloned in to yeast expression plasmid pYES2 using primers FNIP1-KpnI-FLAG-F/FNIP1-XhoI-R and FNIP2-KpnI-FLAG-F/FNIP2-XhoI-R.

Yeast growth media. Yeast cells were grown on YPDA $(2 \%(\mathrm{w} / \mathrm{v})$ Bacto peptone, $1 \%$ yeast extract, $2 \%$ glucose and $20 \mathrm{mg}$ per litre adenine) and YPGal (2\% (w/v) Bacto peptone, $1 \%$ yeast extract, $2 \%$ galactose and $20 \mathrm{mg}$ per litre adenine). Selective growth was on dropout $2 \%$ glucose (DO) medium with appropriate amino acids $^{30}$. Medium $\mathrm{pH}$ was adjusted to 6.8 with $\mathrm{NaOH}$ before autoclaving. The yeast strain pp30 (MAT a, trp1-289, leu2-3,112, his3-200, ura3-52, ade2-101, lys2-801, hsc82KANMX4 and hsp82KANMX4) expressing Hsp82-Ycplac111 or Hsp90 $\alpha$ Ycplac111 as the sole Hsp90 were used in this study. These yeast strains were reported previously by Mollapour et al. ${ }^{31}$. Glutathione $S$-transferase (GST)-tagged Lst 4 under control of the GAL1/10 promoter expressed in a BY4741 (MATa) background was acquired from GE Dharmacon.

Mammalian cell culture. Cultured cell lines human embryonic kidney (HEK293) grown in DMEM (Sigma-Aldrich), human bladder (T24) and human colorectal adenocarcinoma (HT29) grown in McCoy's 5a medium (Sigma-Aldrich), human prostate cancer (LNCaP) and human non-small cell lung cancer (H1299) grown in RPMI 1640 medium (Sigma-Aldrich) and human breast adenocarcinoma (MCF7) grown in Eagle's minimum essential medium (Sigma-Aldrich). Cultured cell lines were acquired from (American Type Culture Collection) and their media was supplemented with $10 \%$ fetal bovine serum (Sigma-Aldrich) and grown in a CellQ incubator (Panasonic Healthcare) at $37^{\circ} \mathrm{C}$ in an atmosphere containing $5 \% \mathrm{CO}_{2}$.

Ex vivo culture and analysis of human RCC tumours. Tumour and adjacent normal tissues of the patients with conventional RCC were obtained with written informed consent from the Department of Urology at SUNY Upstate Medical University. At the time of radical or partial nephrectomy, which was done with $<10 \mathrm{~min}$ of renal ischaemia, RCC tumours were dissected into $\sim 3 \mathrm{~mm}^{3}$ pieces and protein was extracted and quantified as previously described in detail ${ }^{22}$.

Bacterial expression and purification of proteins. All proteins were expressed in Escherichia coli strain BL21 (DE3) and included an N-terminal $6 \times$ His tag. Purification buffers included 20-50 mM Tris or phosphate $\mathrm{pH} 8.0$ and $10 \mathrm{mM} \beta$-mercaptoethanol. Chromatography resins were purchased from GE Healthcare Bio-Sciences (Marlborough, MA), except for Ni-NTA agarose, which was purchased from Qiagen (Valencia, CA). Transformed cells were grown at $37^{\circ} \mathrm{C}$ in lysogeny broth (LB) with $50 \mathrm{mgl}^{-1}$ ampicillin until $\mathrm{OD}_{600}=0.6$. For Hsp90 $\alpha$, cultures were then cooled to $20^{\circ} \mathrm{C}$ and induced with $20 \mathrm{mgl}^{-1}$ isopropyl- $\beta$-D-thiogalactoside overnight. Cells were harvested by centrifugation and lysed enzymatically. Hsp90 $\alpha$ expressed in the supernatant and was isolated by sequential Ni-NTA metal affinity (10-250 mM imidazole step gradient), Q-Sepharose anion exchange (0-1 M NaCl gradient) and Superdex-75 size-exclusion chromatography. Purified Hsp90 $\alpha$ was nucleotide free evidenced by an $A_{280 / 260}$ ratio of 1.83 . For FNIP1-D, cells were induced with $20 \mathrm{mgl}^{-1}$ isopropyl- $\beta$-D-thiogalactoside at $37^{\circ} \mathrm{C}$ for $3 \mathrm{~h}$, harvested by centrifugation and lysed by sonication. FNIP1-D expressed in inclusion bodies and was purified by washing the pellet $3 \times$ in buffer, dissolving the pellet in $8 \mathrm{M}$ urea, subjecting the dissolved pellet to Ni-NTA metal affinity chromatography (10-250 $\mathrm{mM}$ imidazole step gradient) and refolding by rapid dilution. Proteins were $>90 \%$ pure. Concentrations were determined using calculated extinction coefficients as previously described ${ }^{19}$. Proteins were flash frozen on dry ice and stored at $-80^{\circ} \mathrm{C}$ until use.

Immunoprecipitation and immunoblotting. For IP, mammalian cell lysates were incubated with anti-FLAG antibody conjugated beads (Sigma) for $2 \mathrm{~h}$ at $4{ }^{\circ} \mathrm{C}$. Pulldowns were achieved by incubating lysate with Ni-NTA agarose (Qiagen) for $2 \mathrm{~h}$ at $4{ }^{\circ} \mathrm{C}$ or with anti-Hsp90 antibody (835-16F1, Enzo Life Sciences), or antiHsp82 (yHsp90), FLCN (Cell Signaling), FNIP1 or FNIP2 (NCI) 
for $1 \mathrm{~h}$ followed by protein $\mathrm{G}$ agarose for $2 \mathrm{~h}$ at $4{ }^{\circ} \mathrm{C}$. Immunopellets were washed four times with fresh lysis buffer $(20 \mathrm{mM}$ HEPES pH 7.0, $100 \mathrm{mM} \mathrm{NaCl}, 1 \mathrm{mM} \mathrm{MgCl}_{2}, 0.1 \% \mathrm{NP} 40$, protease inhibitor cocktail (Roche) and PhosSTOP (Roche). Proteins bound to Ni-NTA agarose were washed with $50 \mathrm{mM}$ imidazole in lysis buffer $(20 \mathrm{mM}$ Tris- $\mathrm{HCl} \mathrm{pH} \mathrm{7.5,100} \mathrm{mM} \mathrm{NaCl}$, protease inhibitor cocktail (Roche) and PhosSTOP (Roche)) and eluted with either $300 \mathrm{mM}$ imidazole in lysis buffer or with $5 \times$ Laemmli buffer. Precipitated proteins were separated by SDS-PAGE and transferred to nitrocellulose membranes. Co-immunoprecipitated proteins were detected by immunoblotting with indicated dilutions of antibodies recognizing 1:8,000 FLAG (Cat. A2220, SigmaAldrich); 1:2,000 Tetra-His (Cat. 34670, Qiagen); 1:8,000 Hsp90835-16F1 (Cat. ADI-SPA-835), 1:10,000 GAPDH (Cat. ADICSA-335), 1:2,000 p23 (Cat. ADI-SPA-670) (ENZO Life Sciences); 1:20,000 Hsp70 (Cat. SPC-103C/D, StressMarq); 1:1,000 CFTR (Cat. 05-583, Millipore); 1:4,000 FLCN (Cat. 3697), 1:2,000 GR (Cat. 12041), 1:2,000 p-GR-S211 (Cat. 4161), 1:2,000 ER $\alpha$ (Cat. 13258), 1:1,000 CHIP (Cat. 2080), 1:2,000 Grp94 (Cat. 2104), 1:2,000 cMyc (Cat. 2276), 1:2,000 HA (Cat. 3724), 1:1,000 CCT2 (Cat. 3661), 1:1,000 ULK1 (Cat. 8054), 1:1,000 PP5 (Cat. 2289), p50 cdc37 (Cat. 4793), 1:2,000 c-Src (Cat. 2123), 1:2,000 p-cSrc-Y416 (Cat. 2101), 1:4,000 Akt (Cat. 2967), 1:2,000 p-Akt-S473 (Cat. 4060) and 1:2,000 p60 Hop (Cat. 5670), cleaved caspase-3 (Asp175) (Cat. 9664), 1:2,000 cleaved caspase-7 (Asp198) (Cat. 9491) and 1:2,000 cleaved PARP (Asp214) (Cat. 9544) (Cell Signalling); 1:4,000 GST (Cat. 374171), 1:4,000 Raf-1 (Cat. sc-133), $1: 4,000$ Cdk4 (Cat. sc-601) and 1:1,000 Ubiquitin (Cat. sc-8017) (Santa Cruz Biotechnology); and 1:1,000 Aha1 (Cat. 600-401-974, Rockland). Antibodies (1:500 FNIP1 and 1:500 FNIP2) were generated at NCI-NIH. Sbal and Hsp82 (yHsp90) antibodies were generated at Institute of Cancer Research, UK. Secondary antibodies raised against goat (Cat. sc-2020), mouse (Cat. sc-2005), rabbit (Cat. sc-2004) and rat (Cat. sc-2006) (Santa Cruz Biotechnology) were used at 1:4,000 dilution. Representative uncropped western blottings are shown in Supplementary Fig. 6.

Protein labelling and $K_{\mathbf{d}}$ measurements. FNIP1-D protein was desalted into $50 \mathrm{mM}$ Tris $\mathrm{pH} 7.2,150 \mathrm{mM} \mathrm{NaCl}$ and $1 \mathrm{mM}$ TCEP using a PD-10 desalting column and labelled with Texas Red C2 maleimide. Labelling stoichiometry was 0.75 labels/FNIP1-D using $\varepsilon_{595}=104,000 \mathrm{M}^{-1} \mathrm{~cm}^{-1}$ for Texas Red and a correction factor of $0.26 \times \varepsilon_{595}$ to account for its absorbance at $280 \mathrm{~nm}$. Hsp90 $\alpha$ at the indicated concentrations was incubated on ice in $50 \mathrm{mM}$ Tris $\mathrm{pH} 7.2,150 \mathrm{mM} \mathrm{NaCl}, 1 \mathrm{mM}$ TCEP, $4 \mathrm{mM} \mathrm{MgCl}_{2}$ with or without $1 \mathrm{mM}$ ATP, ADP or AMPPNP for $10 \mathrm{~min}$, then incubated with $1 \mu \mathrm{M}$ labelled FNIP1-D for $30 \mathrm{~min}$. Fluorescence anisotropy was measured using a SpectraMax i3 equipped with rhodamine fluorescence polarization module $\left(\lambda_{\mathrm{ex}} \backslash \lambda_{\mathrm{em}}=535 \mathrm{~nm} /\right.$ $595 \mathrm{~nm}$ ). Curve fitting was done in KaleidaGraph 4.0.

MS analysis. Visible bands were excised from the gel manually and cut into small pieces $\sim 1 \mathrm{~mm} \times 1 \mathrm{~mm}$. These gel pieces were destained using 1:1 30-mM potassium ferricyanide:100-mM sodium thiosulfate for $10 \mathrm{~min}$. The destained gel pieces were then washed with $25 \mathrm{mM}$ ammonium bicarbonate and acetonitrile alternatively for $5 \mathrm{~min}$ each wash. This cycle of $5 \mathrm{~min} 25 \mathrm{mM}$ ammonium bicarbonate wash followed by $5 \mathrm{~min}$ acetonitrile wash was repeated three times. To further prepare the gel pieces for digestion, the gel pieces were then dehydrated in $100 \%$ acetonitrile. After removing all acetonitrile, $25 \mu \mathrm{l}$ of porcine trypsin (Promega) dissolved in $25 \mathrm{mM}$ ammonium bicarbonate at a concentration of $20 \mu \mathrm{g} \mathrm{ml}^{-1}$ was added to the gel pieces. The gel pieces were then kept at room temperature overnight $(\sim 12-16 \mathrm{~h})$. Following digestion, the supernatant was transferred to a second tube and acetonitrile was added to the gel pieces to complete the extraction of digested peptides. This extract was added to the first supernatant and this combined solution, containing the extracted peptides, was frozen and lyophilized. The peptides were resuspended in $5 \mu$ l of 100:99:1 acetonitrile:water:trifluoroacetic acid immediately before spotting on the matrix-assisted laser desorption/ionization (MALDI) target.

For MALDI analysis, the matrix solution consisted of $\alpha$-cyano4-hydroxycinnamic acid (Aldrich Chemical Co., Milwaukee, WI) saturating a solution of 1:1:0.01 acetonitrile: $25 \mathrm{mM}$ ammonium citrate:trifuoroacetic acid. Approximately $0.15 \mu \mathrm{l}$ of peptide solution was spotted on the MALDI target immediately followed by $0.15 \mu \mathrm{l}$ of the matrix solution. This combined solution was allowed to dry at room temperature. MALDI-MS and MS/MS data were then acquired using the ABSCIEX TOF/TOF 5800 Mass Spectrometer. Resultant peptide mass fingerprint and peptide sequence data were submitted to the UniProt database using the Mascot search engine to which relevance is calculated and scores are displayed.

Hsp90 ATPase activity in vivo. ATPase activity of human Hsp $90 \propto$ isolated from prostate cancer PC3 cells and its activation by Aha1 or inhibition by HA-FNIP1, HA-FNIP1-D and HAFNIP2 from HEK293 cells were measured as previously described $^{19}$. Cells were transiently transfected with Hsp90 $\alpha-\mathrm{HA}$ (PC3) or the Aha1-FLAG, HA-FNIP1, HA-FNIP1-D and HA-FNIP2 constructs (HEK293). Following protein extraction and IP, protein-bound HA (Sigma) or FLAG (Sigma) affinity beads were washed five times in $0.5 \mathrm{M} \mathrm{NaCl}$ and $1 \% \mathrm{NP} 40$ buffer. Proteins were competed off the beads with either HA or FLAG (Sigma) peptide, at $4{ }^{\circ} \mathrm{C}$ for $2 \mathrm{~h}$ with agitation. Protein was then concentrated with Amicon Ultra- $2 \mathrm{ml}, 10 \mathrm{~K}$ centrifugal filters (Millipore). Using the Micro BCA Protein Assay Kit (Thermo Scientific), protein was quantified and $1 \mu \mathrm{g}$ was run on an SDSPAGE gel as described previously, to standardize the amount of protein used in the assay. Assay was performed as described in the $\mathrm{P}_{\mathrm{i}} \mathrm{Per}$ Phosphate Assay Kit instructions for use (Life Technologies). Standard curve with linear fit line was created from 0 to $100 \mu \mathrm{M}$ final concentration reactions. Hsp90 $\alpha(2.5 \mu \mathrm{g})$ and $5 \mu \mathrm{g}$ of Aha1, FNIP1, FNIP1-D and FNIP2 were added to each reaction run in triplicate, incubated at $37^{\circ} \mathrm{C}$ for $1 \mathrm{~h}$, with $1 \mathrm{mM}$ ATP as substrate and $10 \mu \mathrm{M}$ GB was used in drug reactions. ATP turnover was calculated as mmol $\mathrm{P}_{\mathrm{i}}$ per mol Hsp $90 \alpha$ per minute and relative ATPase activity was calculated from those values, with the value of $\mathrm{Hsp} 90 \alpha$ alone representing $100 \%$ activity.

Statistical analysis. The data presented are the representative or examples of three biological replicates, unless it is specified. Data were analysed with unpaired $t$-test. Asterisks in figures indicate significant differences $\left({ }^{\star} P<0.05,{ }^{* *} P<0.005,{ }^{* * *} P<0.0005\right.$ and $\left.{ }_{* * * *} P<0.0001\right)$. Error bars represent the s.d. or s.e. for three independent experiments, unless it is indicated.

Data availability. The authors declare that the data supporting the findings of this study are available within the article and its Supplementary Information files.

\section{References}

1. Rohl, A., Rohrberg, J. \& Buchner, J. The chaperone Hsp90: changing partners for demanding clients. Trends Biochem. Sci. 38, 253-262 (2013).

2. Neckers, L. \& Workman, P. Hsp90 molecular chaperone inhibitors: are we there yet? Clin. Cancer Res. 18, 64-76 (2012).

3. Panaretou, B. et al. ATP binding and hydrolysis are essential to the function of the Hsp90 molecular chaperone in vivo. EMBO J. 17, 4829-4836 (1998). 
4. Cox, M. B. \& Johnson, J. L. The role of p23, Hop, immunophilins, and other co-chaperones in regulating Hsp90 function. Methods Mol. Biol. 787, 45-66 (2011).

5. Li, J., Richter, K., Reinstein, J. \& Buchner, J. Integration of the accelerator Aha1 in the Hsp90 co-chaperone cycle. Nat. Struct. Mol. Biol. 20, 326-331 (2013).

6. Panaretou, B. et al. Activation of the ATPase activity of hsp90 by the stressregulated cochaperone aha1. Mol. Cell 10, 1307-1318 (2002).

7. Schmidt, L. S. \& Linehan, W. M. Molecular genetics and clinical features of Birt-Hogg-Dube syndrome. Nat. Rev. Urol. 12, 558-569 (2015).

8. Baba, M. et al. Folliculin encoded by the BHD gene interacts with a binding protein, FNIP1, and AMPK, and is involved in AMPK and mTOR signaling. Proc. Natl. Acad. Sci. USA 103, 15552-15557 (2006).

9. Hasumi, H. et al. Folliculin-interacting proteins Fnip1 and Fnip2 play critical roles in kidney tumor suppression in cooperation with Flcn. Proc. Natl. Acad. Sci. USA 112, E1624-E1631 (2015).

10. Hasumi, H. et al. Identification and characterization of a novel folliculininteracting protein FNIP2. Gene 415, 60-67 (2008).

11. Li, X. et al. Analogs of the Allosteric Heat Shock Protein 70 (Hsp70) Inhibitor, MKT-077, as Anti-Cancer Agents. ACS Med. Chem. Lett. 4 (2013).

12. Rohl, A. et al. Hsp90 regulates the dynamics of its cochaperone Stil and the transfer of Hsp70 between modules. Nat. Commun. 6, 6655 (2015).

13. Ying, W. et al. Ganetespib, a unique triazolone-containing Hsp90 inhibitor, exhibits potent antitumor activity and a superior safety profile for cancer therapy. Mol. Cancer Ther. 11, 475-484 (2012).

14. Barrott, J. J. et al. Optical and radioiodinated tethered Hsp90 inhibitors reveal selective internalization of ectopic Hsp90 in malignant breast tumor cells. Chem. Biol. 20, 1187-1197 (2013).

15. Caldas-Lopes, E. et al. Hsp90 inhibitor PU-H71, a multimodal inhibitor of malignancy, induces complete responses in triple-negative breast cancer models. Proc. Natl. Acad. Sci. USA 106, 8368-8373 (2009).

16. Xu, Y., Singer, M. A. \& Lindquist, S. Maturation of the tyrosine kinase c-src as a kinase and as a substrate depends on the molecular chaperone Hsp90. Proc. Natl. Acad. Sci. USA 96, 109-114 (1999).

17. Youker, R. T., Walsh, P., Beilharz, T., Lithgow, T. \& Brodsky, J. L. Distinct roles for the Hsp40 and Hsp90 molecular chaperones during cystic fibrosis transmembrane conductance regulator degradation in yeast. Mol. Biol. Cell 15, 4787-4797 (2004).

18. Wang, X. et al. Hsp90 cochaperone Aha1 downregulation rescues misfolding of CFTR in cystic fibrosis. Cell 127, 803-815 (2006).

19. Dunn, D. M. et al. c-Abl mediated tyrosine phosphorylation of Ahal activates its co-chaperone function in cancer cells. Cell Rep. 12, 1006-1018 (2015).

20. Levine, T. P., Daniels, R. D., Gatta, A. T., Wong, L. H. \& Hayes, M. J. The product of C9orf72, a gene strongly implicated in neurodegeneration, is structurally related to DENN Rab-GEFs. Bioinformatics 29, 499-503 (2013).

21. Beebe, K. et al. Posttranslational modification and conformational state of heat shock protein 90 differentially affect binding of chemically diverse small molecule inhibitors. Oncotarget 4, 1065-1074 (2013).

22. Woodford, M. R. et al. Mps1 mediated phosphorylation of Hsp90 confers renal cell carcinoma sensitivity and selectivity to Hsp90 inhibitors. Cell Rep. 14, 872-884 (2016)

23. Linehan, W. M. \& Ricketts, C. J. Decade in review-kidney cancer: discoveries, therapies and opportunities. Nat. Rev. Urol. 11, 614-616 (2014).

24. Nickerson, M. L. et al. Mutations in a novel gene lead to kidney tumors, lung wall defects, and benign tumors of the hair follicle in patients with the BirtHogg-Dube syndrome. Cancer Cell 2, 157-164 (2002).

25. Nahorski, M. S. et al. Birt Hogg-Dube syndrome-associated FLCN mutations disrupt protein stability. Hum. Mutat. 32, 921-929 (2011).

26. Takagi, Y. et al. Interaction of folliculin (Birt-Hogg-Dube gene product) with a novel Fnip1-like (FnipL/Fnip2) protein. Oncogene 27, 5339-5347 (2008).
27. Mollapour, M. et al. Asymmetric Hsp90 N domain SUMOylation recruits Aha1 and ATP-competitive inhibitors. Mol. Cell 53, 317-329 (2014).

28. Walton-Diaz, A., Khan, S., Bourboulia, D., Trepel, J. B., Neckers, L. \& Mollapour, M. Contributions of co-chaperones and post-translational modifications towards Hsp90 drug sensitivity. Future Med. Chem. 5, 1059-1071 (2013).

29. Holmes, J. L., Sharp, S. Y., Hobbs, S. \& Workman, P. Silencing of HSP90 cochaperone AHA1 expression decreases client protein activation and increases cellular sensitivity to the HSP90 inhibitor 17-allylamino-17-demethoxygeldanamycin. Cancer Res. 68, 1188-1197 (2008).

30. Adams, A., Gottschling, D. E., Kaiser, C. A. \& Stearns, T. Methods in Yeast Genetics (Cold Spring Harbor Laboratory Press, 1997).

31. Mollapour, M. et al. Threonine 22 phosphorylation attenuates hsp90 interaction with cochaperones and affects its chaperone activity. Mol. Cell $\mathbf{4 1}$, 672-681 (2011).

\section{Acknowledgements}

We thank Dr Len Neckers (NCI), Dr Jane B. Trepel (NCI) and Dr Masaya Baba (Kumamoto University) for their constructive comments. We are grateful to Dr J.L. Brodsky (University of Pittsburgh) for CFTR plasmid, Dr Weiwen Ying (Synta Pharmaceuticals) for GB, Dr Gabriela Chiosis (Memorial Sloan Kettering Cancer Center) for PU-H71 and Dr Jason Gestwicki for JG-98 (University of California San Francisco). This work was partly supported by the Intramural Research Program of the NIH, National Cancer Institute, Center for Cancer Research; Frederick National Laboratory for Cance Research, NIH, under contract HHSN261200800001E, DoD W81XWH-15-1-0072 and Wellcome Trust $095605 / Z 11 / Z$. This research was fully supported with funds from the SUNY Upstate Medical University, Upstate Foundation, One Square Mile of Hope Foundation and Carol M. Baldwin Breast Cancer Fund.

\section{Author contributions}

M.R.W., D.M.D., A.R.B., D.C., D.L., B.P., C.P., P.F.H., A.S. and S.N.L. performed experiments. D.B., T.A.H., W.M.L., L.S.S., G.B. and M.M. designed experiments. M.R.W., W.A. and M.M. wrote the manuscript. M.M. conceived the project.

\section{Additional information}

Supplementary Information accompanies this paper at http://www.nature.com/ naturecommunications

Competing financial interests: The authors declare no competing financial interests.

Reprints and permission information is available online at http://npg.nature.com/ reprintsandpermissions/

How to cite this article: Woodford, M. R. et al. The FNIP co-chaperones decelerate the Hsp90 chaperone cycle and enhance drug binding. Nat. Commun. 7:12037 doi: 10.1038/ ncomms12037 (2016).

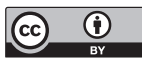

This work is licensed under a Creative Commons Attribution 4.0 International License. The images or other third party material in this article are included in the article's Creative Commons license, unless indicated otherwise in the credit line; if the material is not included under the Creative Commons license, users will need to obtain permission from the license holder to reproduce the material To view a copy of this license, visit http://creativecommons.org/licenses/by/4.0/

(C) The Author(s) 2016 